\title{
An ALMA survey of the SCUBA-2 Cosmology Legacy Survey UKIDSS/UDS field: Source catalogue and properties
}

\author{
Stuart M. Stach ${ }^{\star \star}$ U. Dudzevičiūtè, ${ }^{1}$ Ian Smail, ${ }^{1}$ A. M. Swinbank, ${ }^{1}$ J. E. Geach, ${ }^{2}$
} J. M. Simpson, ${ }^{3}$ Fang Xia An, ${ }^{4,1}$ Omar Almaini, ${ }^{5}$ Vinodiran Arumugam, 6,7 A. W. Blain, ${ }^{8}$ S. C. Chapman, ${ }^{9}$ Chian-Chou Chen, ${ }^{6}$ C. J. Conselice, ${ }^{10}$ E. A. Cooke, ${ }^{1}$ K. E. K. Coppin, ${ }^{2}$ E. da Cunha, ${ }^{11}$ J. S. Dunlop, ${ }^{7}$ Duncan Farrah,, ${ }^{12,13}$ B. Gullberg, ${ }^{1}$ J. A. Hodge, ${ }^{14}$ R. J. Ivison, ${ }^{6,7}$ Dale D. Kocevski, ${ }^{15}$ M. J. Michałowski, ${ }^{16}$ Takamitsu Miyaji, ${ }^{17}$ Douglas Scott A. P. Thomson, ${ }^{19}$ J. L. Wardlow, ${ }^{20}$ Axel Weiss, ${ }^{21}$ P. van der Werf ${ }^{14}$

${ }^{1}$ Centre for Extragalactic Astronomy, Department of Physics, Durham University, Durham, DH1 3LE, UK

${ }^{2}$ Centre for Astrophysics Research, School of Physics, Astronomy and Mathematics, University of Hertfordshire, Hatfield AL10 $9 A B$, UK

${ }^{3}$ Academia Sinica Institute of Astronomy and Astrophysics, No. 1, Sec. 4, Roosevelt Rd., Taipei 10617, Taiwan

${ }^{4}$ Purple Mountain Observatory, China Academy of Sciences, 2 West Beijing Road, Nanjing 210008, China

${ }^{5}$ School of Physics and Astronomy, University of Nottingham, University Park, Nottingham, NG7 2RD, UK

${ }^{6}$ European Southern Observatory, Karl Schwarzschild Strasse 2, Garching, Germany

${ }^{7}$ Institute for Astronomy, University of Edinburgh, Royal Observatory, Blackford Hill, Edinburgh EH9 3HJ, UK

${ }^{8}$ Department of Physics and Astronomy, University of Leicester, University Road, Leicester LE1 7RH, UK

${ }^{9}$ Department of Physics and Atmospheric Science, Dalhousie University Halifax, NS B3H 3J5, Canada

${ }^{10}$ School of Physics and Astronomy, University of Nottingham, University Park, Nottingham, NG7 2RD, UK

${ }^{11}$ Research School of Astronomy and Astrophysics, The Australian National University, Canberra ACT 2611, Australia

${ }^{12}$ Department of Physics and Astronomy, University of Hawaii, 2505 Correa Road, Honolulu, HI 96822, USA

${ }^{13}$ Institute for Astronomy, 2680 Woodlawn Drive, University of Hawaii, Honolulu, HI 96822, USA

${ }^{14}$ Leiden Observatory, Leiden University, P.O. box 9513, NL-2300 RA Leiden, The Netherlands

${ }^{15}$ Department of Physics and Astronomy, Colby College, Waterville, ME 04961, USA

${ }^{16}$ Astronomical Observatory Institute, Faculty of Physics, Adam Mickiewicz University, ul. Stoneczna 36, 60-286 Poznań, Poland

${ }^{17}$ Instututo de Astronomía sede Ensenada, Universidad Nacional Autónoma de México, Ensenada, 22860, México

${ }^{18}$ Department of Physics and Astronomy, University of British Columbia, 6224 Agricultural Road, Vancouver, BC V6T 1Z1, Canada

${ }^{19}$ The University of Manchester, Oxford Road, Manchester, M13 9PL, UK

${ }^{20}$ Department of Physics, Lancaster University, Lancaster, LA1 $4 Y B$, UK

${ }^{21}$ Max-Planck-Institut für Radioastronomie, Auf dem Hügel 69 D-53121 Bonn, Germany

8 March 2019

\begin{abstract}
We present the catalogue and properties of sources in AS2UDS, an 870- $\mu \mathrm{m}$ continuum survey with the Atacama Large Millimetre/sub-millimetre Array (ALMA) of 716 single-dish sub-millimetre sources detected in the UKIDSS/UDS field by the SCUBA2 Cosmology Legacy Survey. In our sensitive ALMA follow-up observations we detect 708 sub-millimetre galaxies (SMGs) at $>4.3 \sigma$ significance across the $\sim 1$-degree diameter field. We combine our precise ALMA positions with the extensive multi-wavelength coverage in the UDS field to fit the spectral energy distributions of our SMGs to derive a median redshift of $z_{\text {phot }}=2.61 \pm 0.09$. This large sample reveals a statistically significant trend of increasing sub-millimetre flux with redshift suggestive of galaxy downsizing. 101 ALMA maps do not show a $>4.3 \sigma$ SMG, but we demonstrate from stacking Herschel SPIRE observations at these positions, that the vast majority of these blank maps correspond to real single-dish sub-millimetre sources. We further show that these blank maps contain an excess of galaxies at $z_{\text {phot }}=1.5-4$ compared to random fields, similar to the redshift range of the ALMA-detected SMGs. In addition, we combine X-ray and mid-infrared active galaxy nuclei activity (AGN) indicators to yield a likely range for the AGN fraction of $8-28 \%$ in our sample. Finally, we compare the redshifts of this population of high-redshift, strongly star-forming galaxies with the inferred formation redshifts of massive, passive galaxies being found out to $z \sim 2$, finding reasonable agreement - in support of an evolutionary connection between these
\end{abstract}

(C) 0000 The Authors

Key words: galaxies:starburst - galaxies:high-redshift - sub-millimetre:galaxies 


\section{INTRODUCTION}

Over twenty years ago the first, deep, sub-millimetre wavelength surveys taken at the James Clerk Maxwell Telescope (JCMT) uncovered a population of sub-millimetre bright galaxies (SMGs - e.g. Smail et al. 1997; Hughes et al. 1998; Barger et al. 1998), which were interpreted as showing some of highest rates of star formation observed in galaxies across the whole history of the Universe. Their sub-millimetre emission originates from the reprocessed ultra-violet starlight that has been absorbed by dust and re-emitted in the restframe far-infrared. This population of highly obscured galaxies are most easily selected at sub-millimetre wavelengths and so are termed 'sub-millimetre galaxies' (SMGs).

The selection of these star-forming galaxies at submillimetre wavelengths has both advantages and disadvantages. A major advantage is the strongly negative $K$ correction at sub-millimetre wavelengths arising from the slope of the Rayleigh-Jeans tail of their far-infrared/submillimetre spectral energy distributions (SED). As a result of this negative $K$-correction, a flux limited sub-millimetre survey provides a uniform selection in terms of far-infrared luminosity (at a fixed dust temperature) for sources across a redshift range of $z=1-6$ (figure 4: Blain et al. 2002). Thus sub-millimetre observations are a very effective means to survey for the most strongly star-forming galaxies in the high redshift Universe. However, a major disadvantage of current single dish observatories operating at sub-millimetre wavelengths is their modest angular resolution, 15-30" FWHM, which is too coarse to allow the counterpart to the submillimetre emission to be easily identified at shorter wavelengths, as several candidate galaxies can be encompassed by the single-dish beam. Hence early attempts to pinpoint the location of SMGs to sub-arcsecond resolutions exploited the FIR-radio correlation (e.g. Ivison et al. 1998; Barger et al. 2000; Ivison et al. 2002; Chapman et al. 2005) to match the sub-millimetre sources to their radio bright counterparts. The limitation of such radio identifications is that the radio waveband does not benefit from a strong negative $K$ correction, so there is a bias against identifying the highest redshift $(z>2.5-3)$ SMGs in the radio images. The difficulties with reliably identifying sub-millimetre source counterparts contributed in part to the slow advance in our understanding of these galaxies in the years following their discovery.

Not withstanding the challenges described above, the first large-scale spectroscopic redshift surveys of radio-identified SMGs (Chapman et al. 2005), and later sub/millimetre interferometrically-selected samples (Smolčić et al. 2012; Danielson et al. 2017), found that these galaxies are typically located at redshifts of $z \sim 2.5$. At these redshifts, the sub-millimetre flux of the sources corresponds to far-infrared luminosities $>10^{12}-10^{13} \mathrm{~L}_{\odot}$, i.e. Ultra-Luminous InfraRed Galaxies (ULIRGs). However, SMGs have volume densities three orders of magnitude greater than comparably luminous local ULIRGs (Chapman et al. 2005). Such high infrared luminosities indicate starformation rates (SFR) of the order $100-1000 \mathrm{M}_{\odot} \mathrm{yr}^{-1}$ (Magnelli et al. 2012; Swinbank et al. 2014), a star-formation rate high enough that within a few dynamical times (a hundred million years) the SMG could form the stellar mass of a massive galaxy $M_{*} \gtrsim 10^{11} \mathrm{M}_{\odot}$. Indeed, constraints on the stellar masses of SMGs have found $M_{*} \sim 10^{11}-10^{12} \mathrm{M}_{\odot}$ (Borys et al. 2005; Hainline et al. 2011; Michałowski et al. 2014) making SMGs some of the most massive galaxies at $z \sim 2$. The space density of these sources and their prodigious star-formation rates means that SMGs contribute $\sim 20 \%$ of the Universal star-formation density between $z=1-4$ (Casey et al. 2013; Swinbank et al. 2014). Being both massive and strongly starforming galaxies in the early Universe, SMGs have been proposed as the progenitors of massive local spheroidal galaxies (e.g. Genzel et al. 2003; Blain et al. 2004; Cimatti et al. 2008; Simpson et al. 2014; Toft et al. 2014; Koprowski et al. 2014; Simpson et al. 2017), potentially following an evolutionary path where, following their ultra-luminous infrared phase, the SMG descendants would display both star-formation and obscured AGN activity, and then appears as a quasi-stellar object (QSO), until the system completely exhausts its supply of gas (Coppin et al. 2008; Simpson et al. 2012).

The major advance in studies of SMGs came with the development of sensitive sub-millimetre interferometers: initially the Sub-Millimeter Array (SMA) (Younger et al. 2008; Wang et al. 2011; Smolčić et al. 2012) and more recently the Atacama Large Millimetre/sub-millimeter Array (ALMA) (Hodge et al. 2013; Simpson et al. 2015a; Hatsukade et al. 2016; Walter et al. 2016; Franco et al. 2018; Hatsukade et al. 2018; Cowie et al. 2018). Interferometric observations, in particular with ALMA, allow us to observe SMGs in the sub-millimetre at spatial resolutions more than an order of magnitude finer than achievable in single-dish surveys and free from confusion - enabling detections of sources to flux densities more than an order of magnitude fainter than the single-dish limits.

Deep, blank-field surveys utilising these interferometers have successfully recovered faint, serendipitously detected sources across arcmin ${ }^{2}$ regions such as in the Hubble UltraDeep Field and GOODS-South (Aravena et al. 2016; Walter et al. 2016; Dunlop et al. 2017; Franco et al. 2018; Hatsukade et al. 2018). These are effective surveys for detecting the fainter examples of the SMG population free from the potential biases from clustering of sources around bright detections. However, the modest field of view of interferometers means that such surveys can only cover small areas and as a result have so far yielded relatively few (10's) of detected sources, with only very few of the brightest examples having $S_{870} \gg 1 \mathrm{mJy}$. To obtain statistically robust samples of the brighter SMGs $\left(S_{870} \gtrsim 1-10 \mathrm{mJy}\right)$, whose properties may be the most distinct from 'normal' star-forming galaxies, we require a hybrid approach - where we exploit the fast mapping speed of single-dish telescopes to identify numbers of these relatively rare sources over the large fields needed to yield large samples - combined with interferometric observations in the same sub-millimetre waveband to allow us to precisely locate the counterparts to the single-dish sources. We first employed this dual-survey approach with the ALMA LABOCA Extended Chandra Deep Field South survey (ALESS) (Hodge et al. 2013; Karim et al. 2013). This was an ALMA Cycle 0 survey of the 122 sub-millimetre sources detected in the LABOCA/APEX single-dish survey of the Extended Chandra Deep Field South (LESS: Weiß et al. 2009) and yielded detections of 126 single-dish sources with deboosted $870 \mu \mathrm{m}$ fluxes $S_{870}>3.6 \mathrm{mJy}$. This survey suggested that some previous single-dish detections were in reality multiple galaxies blended by the coarse resolution 
of the single-dish telescope (Karim et al. 2013) and previous multiwavelength methods of identification of SMGs, were failing to correctly locate the counterpart to the submillimetre emission almost half of the time (Hodge et al. 2013; Simpson et al. 2015a,b).

These initial ALMA studies of flux-limited samples have begun to illuminate the range of characteristics of bright sub-millimetre galaxies, free from the selection biases which influenced earlier radio and mid-infrared based studies. In particular they have highlighted the $\sim 10-20 \%$ of the population which are effectively undetectable in even the deepest optical/near-infrared (Simpson et al. 2014), which represent either the highest redshift, the least massive or the most obscured examples of this population. However, the first ALMA surveys lacked the sample size to identify statistically significant subsets of the rarest classes of SMGs. For example there are only two $z \sim 4.4$ [CII]-selected sources in the ALESS survey (Swinbank et al. 2012; Gullberg et al. 2018), which provide an insight into the properties of the more distant examples of sub-millimetre galaxies. Similarly, ALESS yielded just ten X-ray detected AGN-SMG systems, which can be used to probe the co-evolution of super-massive black holes in strongly star-forming galaxies (Wang et al. 2013). To improve the statistical strength of the conclusions about these rarer subclasses of sub-millimetre galaxies, larger surveys are needed in extragalactic survey fields with the deepest supporting data necessary to detect the faintest examples of this population.

Driven by this need, we have just completed a larger study, nearly an order-of-magnitude larger than ALESS, which exploits the wide-field sub-millimetre mapping of key extragalactic survey fields undertaken by the SCUBA-2 Cosmology Legacy Survey (S2CLS: Geach et al. 2017). We focus in this project on the S2CLS $850-\mu \mathrm{m}$ map of the $\sim 1$ degree diameter UKIDSS Ultra Deep Survey (UDS) Field, which was the largest, uniform area mapped by S2CLS. The S2CLS UDS map has a median sensitivity of $\sigma_{850}=0.9$ mJy over an area of 0.96 degrees $^{2}$, with 716 sources catalogued above a $4-\sigma$ detection limit (corresponding to a $2 \%$ false positive rate) of $S_{850} \sim 3.5 \mathrm{mJy}$. We began our investigation of this sample with a pilot ALMA study of a subset of thirty bright SCUBA-2 detected sources in Cycle 1 (Simpson et al. 2015a,b, 2017). We then expanded the study during Cycles 3,4 , and 5 to complete the ALMA $870-\mu \mathrm{m}$ observations of all $716>4 \sigma$ sources. This yields AS2UDS - the ALMA SCUBA-2 UDS survey - the largest, homogeneously selected, sample of SMGs to date with 708 detections, a five-fold increase over the previous largest similarly robust sample. The first results from this survey have already been presented: number counts and rates of multiplicity (Stach et al. 2018), the serendipitous detection of high redshift [CII] emitters (Cooke et al. 2018), and the use of this survey as a training set for machine learning algorithms to identify the multiwavelength counterparts to single-dish submillimetre sources (An et al. 2018). We present a full analysis of the multiwavelength properties of this sample in Dudzevičiūte et al. (in prep.) and in Gullberg et al. (in prep.) we discuss the information available on the sizes and morphologies of the dust continuum in these sources from our highest resolution ALMA observations.

In this paper we present the final catalogue for the
AS2UDS survey ${ }^{1}$. In our analysis we compare results from our new large sample to previous studies. To simplify these comparisons we have limited them in general to flux-limited samples from: 1. larger unbiased blank-field surveys at $850 \mu \mathrm{m}$ (as there is evidence of differences compared to populations selected in the far-infrared and millimetre, e.g. Smolčić et al. 2012; Koprowski et al. 2014; Scudder et al. 2016; Ikarashi et al. 2017); 2. with deep (<1 mJy rms) interferometric identifications in the same waveband as any initial single-dish selection, if appropriate (c.f. Barger et al. 2014; Umehata et al. 2014; Hill et al. 2018); 3. and which are not explicitly lensed, owing to the potential selection effects and variable flux limits as well as uncertainties from cluster lenses and especially galaxy-scale lensed samples (e.g. Weiß et al. 2013; Fujimoto et al. 2016; Arancibia et al. 2018). Thus most of our comparisons are made to the ALESS survey (Hodge et al. 2013), SuperGOODS (Cowie et al. 2018) and the various ALMA surveys in GOODS-S (Walter et al. 2016; Dunlop et al. 2017; Franco et al. 2018; Hatsukade et al. 2018).

In $\S 2$ we describe the target selection for our ALMA survey and data reduction across the different ALMA Cycles and the wealth of multi-wavelength archival data available in this field, most notably from the UKIDSS UDS DR11 catalogue (O. Almaini et al. in prep.). §3 describes the source detection algorithm, the simulated maps used for estimating completeness and flux boosting derivations. In $\S 4$ we present the first results from our MAGPHYS SED fitting (Dudzevičiūtè et al. in prep.): the photometric redshift distribution of our sample and comparisons with previous surveys. In addition we present the selection of active galactic nuclei (AGNs) from our catalogue through archival X-ray observations of the field and IRAC colour-colour selection. $\S 5$ presents our main conclusions. We assume a cosmology with $\Omega_{\mathrm{m}}=0.3, \Omega_{\Lambda}=0.7$, and $\mathrm{H}_{0}=70 \mathrm{~km} \mathrm{~s}^{-1} \mathrm{Mpc}^{-1}$. All magnitudes are in the $\mathrm{AB}$ system and errors are calculated from bootstrap analysis unless otherwise stated.

\section{OBSERVATIONS AND DATA REDUCTION}

\subsection{Sample Selection}

The ALMA-SCUBA-2 Ultra Deep Survey, hereafter AS2UDS, is a high-resolution, sub-millimetre interferometric follow-up survey of the SCUBA-2 $850 \mu \mathrm{m}$ sources selected from the S2CLS map of the UDS field (Fig 1). The parent single-dish survey covers an area of $0.96 \mathrm{deg}^{2}$, with noise levels below $1.3 \mathrm{mJy}$ and a median depth of $\sigma_{850}=0.88$ mJy beam $^{-1}$ with $80 \%$ of sources identified in regions of the map with $\sigma_{850}=0.86-1.02 \mathrm{mJy}$ beam $^{-1}$ (Geach et al. 2017). Across four ALMA cycles (1, 3, 4, and 5) we observed all $716>4 \sigma$ sources from the SCUBA-2 S2CLS map (corresponding to observed flux densities $S_{850} \geq 3.4 \mathrm{mJy}$ ) (see: Figure 1).

In Cycle 1 (Project ID: 2012.1.00090.S), 30 of the brightest sources from an early version of the SCUBA2 UDS map (data taken before 2013 February) were observed in ALMA Band 7 (Simpson et al. 2015a,b, 2017). This early version of the SCUBA-2 map had a depth of

\footnotetext{
1 Catalogue can be found at http://astro.dur.ac.uk/AS2UDS
} 


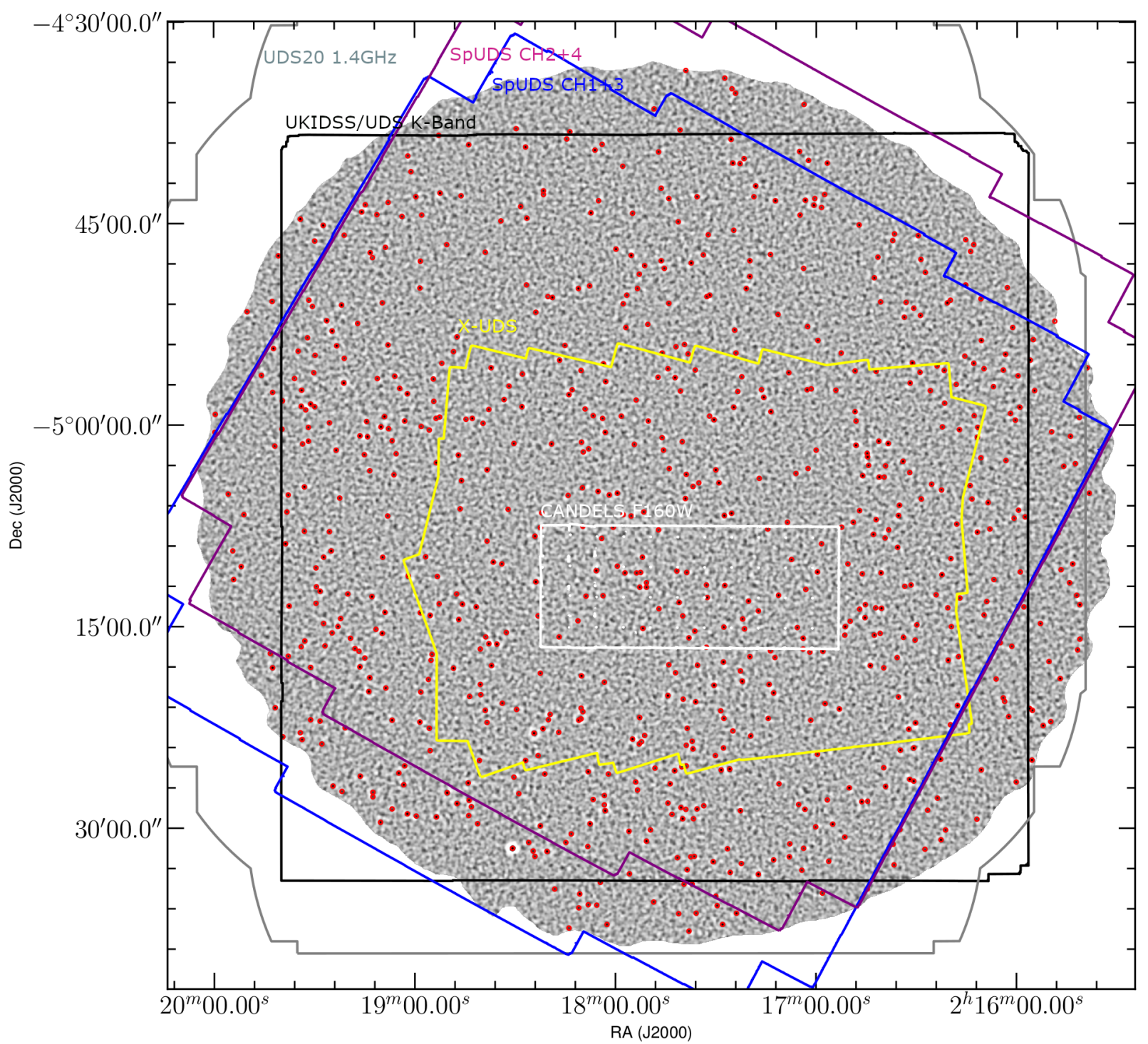

Figure 1. A sample of the multi-wavelength coverage of the AS2UDS sample which motivates this extra-galactic field being chosen for high resolution ALMA follow-up. The background shows the S2CLS $850 \mu \mathrm{m}$ UDS map from which the parent sample is extracted (Geach et al. 2017). The red circles are the primary beams for AS2UDS targetting the $4 \sigma$ detections in the S2CLS map. The black outline shows the $K$-band coverage that forms the footprint for the UKIDSS UDS catalogue (O. Almaini et al. in prep.). The Spitzer/IRAC CH1/3 and $\mathrm{CH} 2 / 4$ coverage is shown in blue/purple respectively, the HST CANDELS F160W in white, VLA 1.4 GHz in grey, and the X-UDS Chandra coverage in yellow (Kocevski et al. 2018).

only $\sigma_{850} \sim 2.0 \mathrm{mJy}^{-1}$ and subsequent integration time in the S2CLS survey scattered three of these sources below our final sample selection criteria $(>4 \sigma)$, leaving 27 of these original single-dish detected sources in our final sample. The remaining 689 single-dish sources in the final S2CLS catalogue were observed across ALMA cycles 3 and 4 (Project ID: 2015.1.01528.S and 2016.1.00434.S, respectively). To cross calibrate the data, a fraction of these sources were observed twice, once in Cycle 3 and 4. In addition, in Cycle 5 ten of the brightest SCUBA-2 sources which returned 'blank' maps from the Cycle 3 and 4 ALMA observations were re-observed at greater depth (Project ID: 2017.1.01492.S), these maps will be discussed further in $\S 4.3$.

\subsection{Data Reduction}

Our ALMA targets were observed in Band 7 (central frequency $344 \mathrm{GHz} \sim 870 \mu \mathrm{m})$. At this frequency the FWHM of the ALMA primary beam (17!'3) covers the FWHM of the SCUBA-2 beam (14." 7 ). Cycle 1 observations were carried out on 2013 November 1 (Simpson et al. 2015a), Cycle 3 between 2016 July 23 and August 11, Cycle 4 between 2016 November 9 and 17 and 2017 May 6, and Cycle 5 on 2018 August 24 .

These ALMA Band 7 continuum observations were 150 second integrations in Cycle 1 using 26 dishes, 40 second integrations in Cycle 3 and 4 (with 45-50 dishes), and 285 second integrations using 44 dishes in Cycle 5, with the 7.5 GHz bandwidth of the four spectral windows centred at 
$344.00 \mathrm{GHz}$. The array configurations for Cycle 1 observations yielded a median synthesised beam size of $0 .{ }^{\prime \prime} 35 \times 0$.' 25 . With Cycle 3 and 4 observations we aimed to match this resolution, however our Cycle 3 observations were taken with a more extended array configurations resulting in median synthesised beam sizes of 0 "' $19 \times 0$ "' 18 ('natural' weighting). Our Cycle 5 observations were intended to test whether the lack of detected counterparts in the ALMA observations of ten SCUBA-2 sources observed in previous cycles was not a result of flux being resolved out, and therefore were taken with a median synthesised beam size of $0 !^{\prime \prime} 81 \times 0$. $^{\prime \prime} 54$.

Phase centres for each observation were set to the SCUBA-2 positions of the S2CLS catalogue at the time of ALMA proposal submissions. For the 689 SCUBA-2 sources followed up in Cycle 3, 4, and 5 this results in phase centres coincident to the SCUBA-2 positions from the S2CLS DR1 sub-millimetre source catalogue (Geach et al. 2017), however for Cycle 1 the 27 SCUBA-2 source positions were not corrected for a systematic astrometric offset in the interim map and an offset exists between the final S2CLS DR1 source position and ALMA phase centres, with a median offset of $3.2_{-0.6}^{+0.1}$ arcsec. The ALMA primary beam size is large enough that this offset still results in the majority $(\sim 95 \%)$ of the SCUBA-2 beam falling within the primary beam of in our 30 Cycle 1 observations and, as discussed below, these are observations which were targeting the brighter SCUBA2 sources and thus we expect detections closer to the offset phase centres and thus still well within the ALMA primary beams.

The Common Astronomy Software Application (CASA McMullin et al. 2007) v4.1, v4.5.3, v4.7.2, v5.3.0 were used to calibrate the datasets from Cycle 1, 3, 4, and 5 respectively using the standard ALMA calibration scripts. For the bandpass and phase calibration observations of J0006-0623, J0423-0120, J0238+1636, J0241-0815, and J0006-0623 were used across the four cycles and for the absolute flux scaling: J0217-0820 and J0238+1636.

For the imaging CASA v 4.7.2 was used, with the CONCAT task to combine the visibilities of the 125 maps that were observed in both Cycle 3 and Cycle 4 . To create the continuum maps we used the CLEAN task in multifrequency synthesis mode. Previous studies on the sizes of sub-millimetre galaxies suggests their sub-millimetre intrinsic sizes are $0{ }^{\prime \prime} 3 \pm 0{ }^{\prime \prime} 1$ (e.g. Simpson et al. 2015b; Hodge et al. 2016; Ikarashi et al. 2017) therefore we expect the majority of our detections to be marginally resolved. To ensure we are not biased against selecting extended sources, by remaining sensitive to extended flux from our SMGs, we employed a $0 . \prime 5$ FWHM Gaussian taper in the $u v$-plane using the uvtaper parameter in CLEAN for the Cycle 1,3, and 4 data. This effectively down-weights the visibilities from the longer baselines thus enhancing the sensitivity to extended emission at the expense of overall reduced sensitivity in the maps. Combined with natural weighting this resulted in 'detection' maps with median synthesized beam sizes of 0 .' $73 \times 0$.' 59 for Cycle 1, $0^{\prime \prime} 56 \times 00^{\prime \prime} 50$ for Cycle 3 and 0 . $^{\prime} 58 \times 0 .{ }^{\prime \prime} 55$ for Cycle 4 (the untapered Cycle 5 maps has beam sizes $0 . \prime 79 \times 0 .{ }^{\prime \prime} 52$ ).

For the ALMA map cleaning, the first step was creating 'dirty' maps, $512 \times 512$ pixels in size with a pixel scale of $0{ }^{\prime \prime} 06$ ( $300^{\prime \prime} 7$ square), by combining the spectral windows with no cleaning cycles applied. We calculated the average root mean square (rms) flux density for each

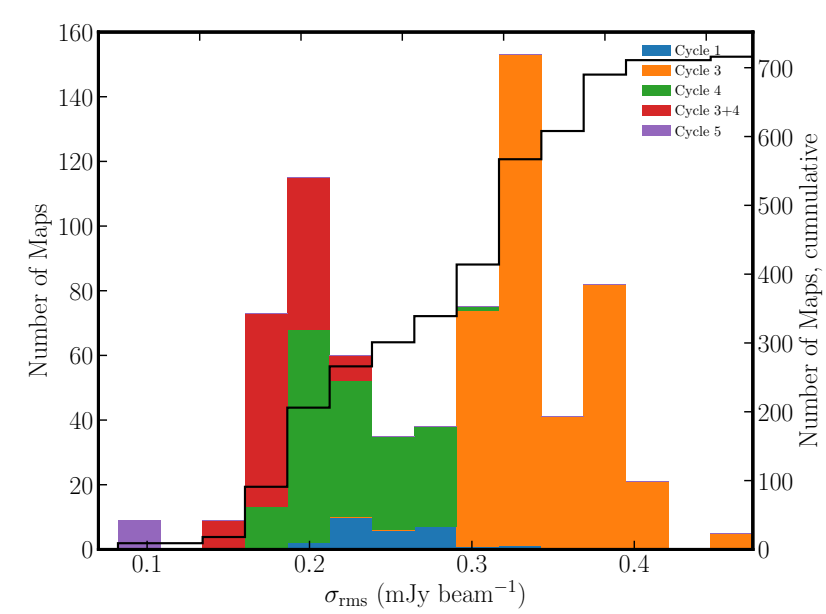

Figure 2. The distribution of the rms noise estimates from the 0 ."5 $u v$-tapered detection maps for the different cycles used in AS2UDS. Adopting similar reconstructed beam sizes across all our maps results in the final rms noise varying inversely with their original observed resolution, with the exception of the purposefully much deeper 'blank' field repeat observations in Cycle 5 . Deviations in rms across the same cycle are a result of different observing conditions. Overlaid is the cumulative distribution of the rms which shows after tapering the median rms of the full survey is $0.3_{-0.2}^{+0.1} \mathrm{mJy}_{\text {beam }}{ }^{-1}$.

of these 'dirty' maps by first removing pixels associated with potential sources in the maps by applying an iterative sigma-clipping routine to the map, and then finding the root mean square value of the unclipped values $(\sigma)$. Each map was then initially cleaned to $3 \sigma$, and islands of pixels with flux densities above $4 \sigma$ were convolved with the reconstructed beam to create cleaning masks. If no islands were detected then the initially-cleaned map was taken as the final map however if any sources were detected then the clean mask was applied and sources cleaned to $1.5 \sigma$. This resulted in final cleaned 'detection' maps which have mean depths of $\sigma_{870}=0.25 \mathrm{mJy}$ beam $^{-1}$ for Cycle 1 , $\sigma_{870}=0.34 \mathrm{mJy}_{\text {beam }^{-1}}$ for Cycle $3, \sigma_{870}=0.23 \mathrm{mJy} \mathrm{beam}^{-1}$ for Cycle 4 , and $\sigma_{870}=0.085 \mathrm{mJy}_{\text {beam }}{ }^{-1}$ for Cycle 5. Figure 2 shows the histogram of the depths for each of the 716 images with the median depth across the survey of $0.3_{-0.2}^{+0.1} \mathrm{mJy}$ beam $^{-1}$, which is $\sim 3 \times$ deeper than the original single-dish SCUBA-2 survey but with a beam $\sim 600 \times$ smaller in area - allowing us to precisely locate the source(s) of the sub-millimetre emission seen by SCUBA-2.

\subsection{Additional Multiwavelength Observations}

In this paper we present some of the basic properties of our sub-millimetre catalogue, using redshifts derived from spectral energy distribution (SED) fitting with MULTIwavelength Analysis of Galaxy Physical Properties (MAGPhys) (Da Cunha et al. 2008). This analysis exploits the wealth of deep, multi-wavelength observations available in this field (e.g. Figure 1). In this section we describe the multi-wavelength observations used in the MAGPHYS analysis, however the full description of the MAGPHYS SED fitting 
and the resulting constraints on the source properties are given in Dudzevičiūtè et al. (in prep.).

The basis of our multi-wavelength analysis is taken from the UKIRT Infrared Deep Sky Survey (UKIDSS) Ultra Deep Survey data release 11 catalogue (O. Almaini et al. in prep.). This survey contains 296,007 $K$-band detected sources, extracted with $2^{\prime \prime}$ diameter apertures corrected to total galaxy magnitudes, detected with SEXTRACTOR with photometry retrieved from $J$ and $H$ maps using SEXTRACTOR dual-image mode. These $J, H$, and $K$-band images were observed through a mosaic of four observations, covering a total survey area of $0.77 \mathrm{deg}^{2}$, with the Wide-Field Camera at UKIRT (Casali et al. 2007), which covers 643 of the 716 pointings of AS2UDS, or $\sim 90 \%$. The DR11 maps achieve a $2^{\prime \prime}$ aperture $3-\sigma$ median depth of $J=26.2, H=25.7$, and $K=25.9$ mag making this one of the deepest near-infrared surveys on degree scales. The DR11 UDS $K$-band selected catalogue has been matched with a number of other surveys to broaden the wavelength coverage, with the matching and photometry measurements described in Hartley et al. (in prep.). We use a $0 .^{\prime \prime} 6$ matching radius to cross-match the DR11 UDS catalogue to our AS2UDS sub-millimetre galaxy catalogue. This matching radius provides a low false match ratio $(\sim 3.5 \%)$ when matching the two catalogues (see: An et al. 2018).

Complementary optical data comes from the Subaru/XMM-Newton deep survey (SXDS) (Furusawa et al. 2008), this is a survey with $B, V, R_{C}, i^{\prime}$ and $z^{\prime}$-band magnitudes with $2^{\prime \prime}$ aperture $3-\sigma$ depths of $B=28.4$, $V=27.8, R_{c}=27.7, i^{\prime}=27.7$ and $z^{\prime}=26.6 \mathrm{mag}$. Additionally there is $Y$-band data with a $3-\sigma$ depth of 25.3 mag supplied from the Visible and Infrared Survey Telescope for Astronomy (VISTA) Deep Extra-galactic Observations (VIDEO) survey (Jarvis et al. 2012).

We include observations in the near-infrared from the Spitzer UKIDSS Ultra Deep Survey (SpUDS; PI: J. Dunlop), a $\sim 1 \mathrm{deg}^{2}$ IRAC (at 3.6, 4.5, 5.8, and $8.0 \mu \mathrm{m}-$ corresponding to channels: Ch1, 2, 3, and 4 respectively) survey of the UDS field with $3-\sigma$ limiting depths of $23.5,23.3$, 22.3 , and $22.4 \mathrm{mag}$ in Ch1-4 respectively. The astrometry of the IRAC images was corrected by stacking the IRAC images on the DR11 UDS $K$-band locations and corrections of +0 .'00 R.A. and +0 .' 15 Dec. were applied to the Ch1 image, +0 "'075 R.A. and +0 .'12 Dec. to Ch2, +0 "'075 R.A. and +0 .' 0 Dec. to Ch3, and +.'6 R.A. -0 .'075 Dec. to Ch4. At each of the AS2UDS galaxy locations 2.'0 aperture corrected magnitudes were measured, in order to be consistent with the other optical photometric bands. We checked the quality of our photometry by comparing our Ch1 and Ch2 aperture corrected magnitudes to those given in the UKIDSS DR11 catalogue (Ch3 and $\mathrm{Ch} 4$ are not supplied in the DR11 catalogue). All sources with a neighbour within 2 .' 5 were checked for possible contamination. Conservatively, we calculated how $K$-band aperture corrected magnitudes (or in some cases $K$-band magnitude limits) of the AS2UDS sources and the near-by sources would change if observed at the resolution of the IRAC Ch1 data. If the flux from a nearby source - as measured in a $2 .^{\prime \prime} 0$ aperture at the position of AS2UDS source - resulted in contamination of over $50 \%$, then IRAC magnitudes were set to limits. This reduced the number of detection in each IRAC band by 111 .

\subsubsection{Far-Infrared}

We include photometry at 100, 160, 250, 350 and $500 \mu \mathrm{m}$, where available, from the Herschel Multi-tiered Extragalactic Survey (HerMES; Oliver et al. 2012). To correct the astrometry of the SPIRE images the same shifts of $<11^{\prime \prime} 5$, found in the AS2UDS pilot sample of Simpson et al. (2017), were applied to the Herschel/SPIRE and PACS images. This shift was derived from re-centering SPIRE stacked images using VLA radio source positions (see $\S 2.3 .3$ ). We have confirmed that the radio astrometry aligns with ALMA to $<0$.' 1 in both R.A. and Dec. and so no additional correction to the SPIRE astrometry is required. SPIRE has comparatively low angular resolution with 18, 25, and 35"FWHM at 250, 350 and $500 \mu \mathrm{m}$ respectively and therefore to deblend the low resolution images we apply the technique described in Swinbank et al. (2014). The flux errors, and the detection limits of $5.2,12.1,9.2,10.6$, and $12.2 \mathrm{mJy}$ at 100, 160, 250, 350 and $500 \mu \mathrm{m}$ respectively are derived from simulations (see: Swinbank et al. 2014).

\subsubsection{Hubble Space Telescope}

A $201.7 \operatorname{arcmin}^{2}$ region of the UDS field (shown in Figure 1) was covered by the Hubble Space Telescope (HST) in the Cosmic Assembly Near-infrared Deep Extragalactic Legacy Survey (CANDELS: Galametz et al. 2013). This provides a WFC3 F160W $\left(H_{160}\right)$ selected catalogue of sources with a 5- $\sigma$ limiting magnitude of 27.45 mag for a point source, with SEXTRACTOR dual-mode source extraction in the F814W $\left(I_{814}\right)$, and F125W $\left(J_{125}\right)$ bands, 47 of our SMGs are covered in the CANDELS region, and we show these in Figure 3.

\subsubsection{Radio}

Imaging at $1.4 \mathrm{GHz}$ is supplied from part of the UDS20 survey (V. Arumugam et al. in prep.) which covers $\sim 1.3 \mathrm{deg}^{2}$, and as can be seen in Figure 1 this mosaic covers the near entirety of the pointings in AS2UDS (714/716 sources). The radio map averages a $1-\sigma$ depth of $10 \mu \mathrm{Jy}_{\text {beam }}{ }^{-1}$ with the deepest regions of the map reaching $7 \mu \mathrm{Jy}$ beam $^{-1}$ with a synthesised beam size of $\sim 1$.' 8 FWHM. We employ a 1 . $^{\prime} 6$ matching radius from the AS2UDS sources to the VLA 4$\sigma$ catalogue given by Arumuham et al. (in prep.) as this is the radius at which the cumulative number of VLA detections flattens, which yields a false matching rate of $1 \%$. For our 708 SMGs, 706 are covered by the UDS20 survey. Of those, 273 have a radio counterpart within 1.'6 (29\%) however this includes close pairs in the AS2UDS catalogue which match to a single radio source therefore only 264 unique radio sources are matched to the AS2UDS catalogue.

\subsubsection{X-ray}

Deep Chandra observations of part of the UDS field have been obtained by the X-UDS survey (Kocevski et al. 2018). This survey covers $0.33 \mathrm{deg}^{2}$ centred around the HST/CANDELS survey region (Figure 1). This coverage comprises a deep centre and shallower coverage over a wider area. In the central $\sim 100 \operatorname{arcmin}^{2}$ of this region, the survey has an average exposure time per pixel of $600 \mathrm{ks}$ and outside 


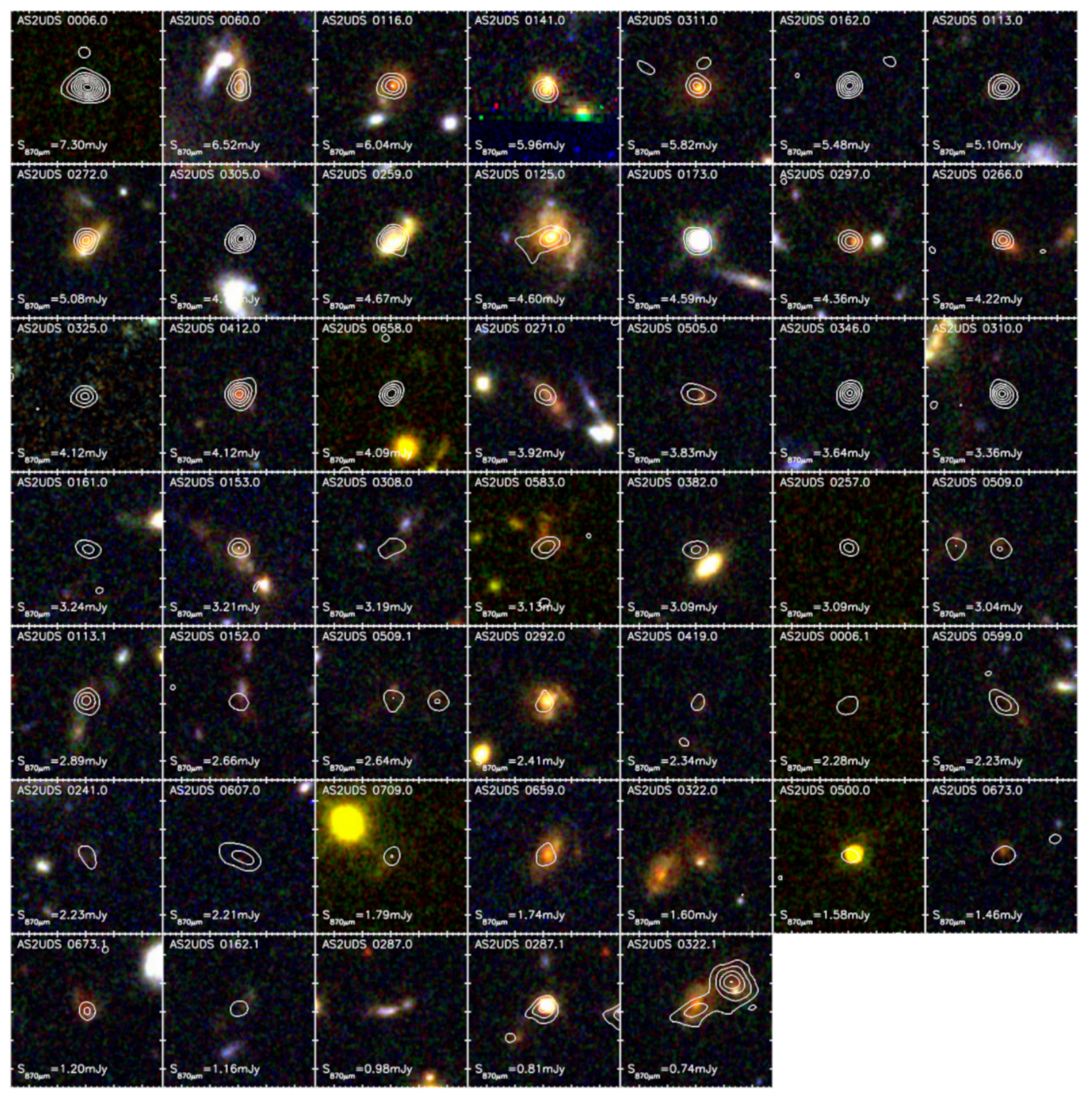

Figure 3. HST $I_{814} J_{125} H_{160}$-band colour images (5".4 square) of the 47 ALMA SMGs in our sample that lie in the CANDELS region. Contours are taken from the tapered ALMA maps, and denote the $870 \mu \mathrm{m}$ emission, and start at $3 \sigma$ and are incremented by $3 \sigma$. The $870 \mu \mathrm{m}$ flux (in mJy) is given in the lower left corner. The HST morphologies of the ALMA SMGs display a range of morphologies, although the majority are morphologically complex. In general the sub-mm emission also appears more compact than the rest-frame optical emission (e.g. Simpson et al. 2015b).

of this area the survey has an exposure of $200 \mathrm{ks}$. In total the X-UDS catalogue has $868 \mathrm{X}$-ray point sources above a flux limit of $4.4 \times 10^{-16} \mathrm{ergs} \mathrm{s}^{-1} \mathrm{~cm}^{-2}$ in the full band $\left(0.5^{-}\right.$ $10 \mathrm{keV})$.

We match the X-UDS catalogue to the AS2UDS catalogue using matching radii based on the positional errors radii in the X-UDS survey (median positional error of 0. '96). Over the full X-UDS coverage we have 274 SMGs falling within the Chandra footprint, of which 21 are matched to X-ray counterparts. Within the CANDELS HST area, where the Chandra coverage is deepest, we have 47 SMGs, but only two of these match to X-UDS sources. We also perform a stacking analysis to derive average X-ray fluxes for samples of individually undetected SMGs. This analysis uses the XUDS Chandra soft $(0.5-2 \mathrm{keV})$ and hard $(2-8 \mathrm{keV})$ band observations and the CSTACK stacking software developed by T. Miyaji. For samples of SMGs we use CSTACK to determine the mean stacked, background subtracted, count-rates and uncertainties from which fluxes were derived using the count rate to flux conversion factors given in Kocevski et al. (2018). These fluxes are then converted into X-ray luminosities by assuming a power-law X-ray spectrum with photon 
index $\Gamma=1.7$, consistent with the SED shape assumed for the X-ray detections in Kocevski et al. (2018), and the photometric redshifts estimated below.

\section{ANALYSIS}

For each ALMA map, 0." 5 diameter aperture noise levels are derived from randomly placing 50 apertures within the area of the primary beam and calculating the standard deviation of the resulting aperture fluxes. This aperture size roughly matches the $u v$-taper size, however, being slightly smaller in area than the resulting ALMA restoring beam sizes, results in $0 . \prime 5$ aperture depths approximately a factor of two deeper than the noise per beams quoted above. We extract sources by initially using SEXTRACTOR to find $>2 \sigma$ peaks in the 'detection' maps. At each of these detections a 0.' 5 diameter aperture flux was measured and, from the aperture noise levels, signal-to-noise ratio (SNR) values calculated for each source.

The AS2UDS catalogue comprises the 708 sources which have $0 .{ }^{\prime \prime} 5$-diameter aperture $\mathrm{SNR} \geq 4.3$. This threshold, as well as the $0 .{ }^{\prime \prime} 5$ aperture size were chosen to maximise the number of sources detected whilst not exceeding a $2 \%$ contamination rate. This is based on the ratio of 'negative' sources found in the inverted ALMA maps (i.e. multiply pixel values by -1 ) compared to the total number of sources detected from both the inverted and regular maps as a function of the signal-to-noise threshold.

To measure the flux density of our sources, 1.'0 diameter aperture fluxes were extracted for the 708 sources from the primary beam corrected maps. A $20 \%$ aperture correction is applied to the fluxes. This correction is derived from aperture fluxes taken from the flux calibrator maps imaged in the same manner as the science targets. We note that our typical sources are not expected to be well-resolved in these $0 . \prime 5$-tapered maps. We discuss the information available on the sizes and morphologies of the AS2UDS SMGs from the untapered higher-resolution observations in Gullberg et al. (in prep.).

\subsection{Completeness and Flux Deboosting}

The completeness of the AS2UDS catalogue and flux deboosting factors were estimated from a suite of 60,000 simulated ALMA maps, as described in Stach et al. (2018). Briefly, the background noise for these maps were taken by selecting ten AS2UDS maps that sampled the $\sigma_{870}$ distribution for the complete survey. The residual maps (the observed ALMA maps minus the flux from any detected sources) for these ten maps were extracted from CASA. One simulated source was then injected into each of these noise maps, to avoid the possibility of multiple injected sources being injected into the same beam location. Following the approach in previous work (Karim et al. 2013; Simpson et al. 2015 b) the fluxes for these sources were randomly sampled from a steeply declining power-law distribution with an index of -2 . The FWHM sizes of the sources were randomly selected from a uniform (and hence conservative) distribution between point sources and 0.'9 FWHM extended sources. These intrinsic sources were then convolved with the ALMA synthesised beam corresponding to the original residual map the simulated source was allocated to, in a random location within the ALMA primary beam. The above described source extraction method was applied to each of the simulated maps and a successful recovery logged when a detection was found within one synthesised beam FWHM, or $0 .{ }^{\prime \prime} 6$ of the injected source position.

The intrinsic sizes of the injected sources has a noticeable effect on the recovered completeness fractions, particularly at lower signal-to-noise (see also: Franco et al. 2018). In Figure 4 we compare the completeness fractions for the recovered sources in Cycle 3, Cycle 4, and Cycle 3+4 for the complete simulated sample and the completeness fractions when cutting simulated sources with intrinsic FWHM $<0$.' 6 . At the highest signal-to-noise, and at the original SCUBA-2 flux limit of $S_{850} \geq 3.6 \mathrm{mJy}$, AS2UDS is $98 \pm 1 \%$ complete for all simulated sources with only the most extended sources suffering some incompleteness (see: Stach et al. 2018). The difference in completeness ratios at the fainter end however becomes more pronounced with a $1.5 \mathrm{mJy}$ source having a median $52 \pm 4 \%$ chance of detection with an intrinsic source size $<0{ }^{\prime \prime} 3,25 \pm 2 \%$ for sizes $0{ }^{\prime \prime} 3--0$. $^{\prime} 6$, and just $7 \pm 1 \%$ for sizes $>0{ }^{\prime \prime} 6$.

Next, we estimate the effect of flux boosting. The flux boosting is a consequence of the tendency of low signal-tonoise ratio sources to have their measured fluxes preferentially increased by noise fluctuations in the maps. We estimate the magnitude of this effect by taking the median ratios of the recovered flux density for each of the detected simulated sources with the known input flux density as a function of the signal-to-noise ratio as shown in Figure 4. As with the completeness calculations, we show that the intrinsic source size distribution can have a noticeable effect on the flux deboosting. In Figure 4 we show the flux recovery as a function of intrinsic source FWHM which indicates that the recovered flux of extended sources is systematically low, this therefore brings the flux deboosting values down. As previous studies suggest median sub-millimetre emission sizes of $\sim 0 .^{\prime \prime} 3$ (Tacconi et al. 2006; Simpson et al. 2015a) we follow Simpson et al. (2015a) and limit our simulated sources to FWHM $<0$ "' 6 . This results in flux deboosting factors of $32 \%$ for sources at our $4.3 \sigma$ detection threshold, reducing to $10 \%$ at $7.8 \sigma$ and $\sim 0 \%$ at $12 \sigma$. A running median is calculated from this ratio as a function of SNR in a $0 .{ }^{\prime \prime} 5$ diameter aperture, shown in Figure. 4, and this median value is used to correct the boosted fluxes to derive our final aperture corrected, flux deboosted, flux densities.

\subsection{SCUBA-2 Positional Offsets}

Surveys such as AS2UDS and ALESS (Hodge et al. 2013) have been motivated by the need to identify large samples of sub-millimetre galaxies to bright single-dish submillimetre sources. In the absence of sub-millimetre interferometry, this requires statistical associations between the single-dish sources and sources identified in higher-resolution multiwavelength observations. These statistical associations are complicated by the significant uncertainties in the singledish source positions. It is expected that these positional uncertainties are dependant on the SNR of the single-dish detection and in Ivison et al. (2007) the ideal theoretical 

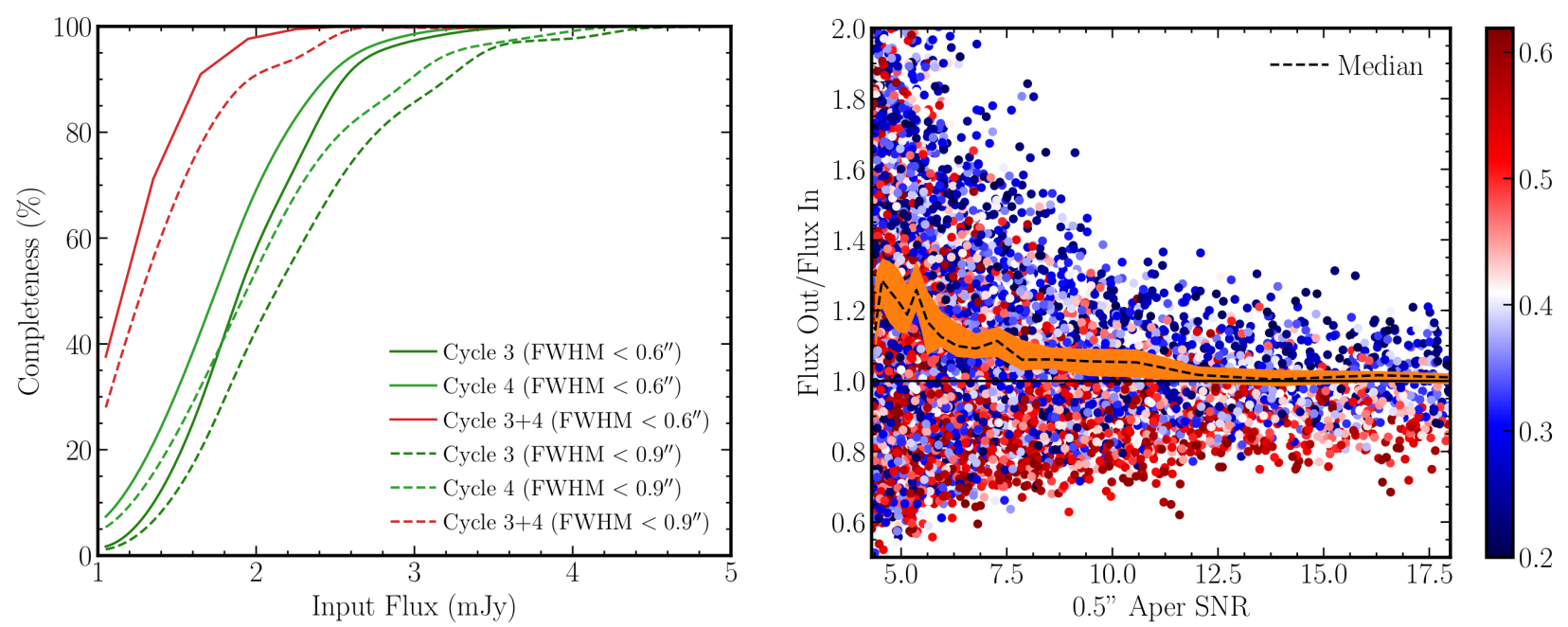

Figure 4. Left: Completeness fractions as a function of the input total flux for each simulated galaxy estimated from 60,000 simulated ALMA observations. As described in Stach et al. (2018) our catalogue is complete for sources above $S_{850} \geq 4$ mJy. However, below this flux limit the completeness declines, reaching $50 \%$ at $1.3 \mathrm{mJy}$ for the ALMA maps that were observed twice. The solid lines show the completeness fractions for simulated sources with intrinsic FWHM $<0$ "' 6 whilst the dashed line shows a more conservative estimate including all simulated sources (with sizes up to 0"9). Right: The ratio of the recovered to injected fluxes for our simulated galaxies showing the influence of flux boosting, where the lower signal-to-noise sources have measured fluxes boosted relatively higher by noise fluctuations. To correct for this we calculate the median boosting (dashed line) with the shaded region showing the $1 \sigma$ bootstrap error. Using the power-law distribution of simulated sources we find a boosting factor of $32 \%$ for sources at our $4.3 \sigma$ threshold. The colour coding of the points is based on the intrinsic FWHM size of the simulated sources using the colour bar on the right (scale in arcseconds). As the extended sources have systematically lower recovered fluxes this tends to reduce the flux deboosting factors.

expression for this dependence was given as:

$\Delta \alpha=\Delta \delta=0.6\left[(\mathrm{SNR})^{2}-(2 \beta+4)\right]^{-1 / 2} \mathrm{FWHM}$,

where $\Delta \alpha$ and $\Delta \delta$ are the rms positional errors in R.A. and Dec., SNR is the signal to noise ratio of the SCUBA-2 detection, FWHM is the full-width-half-maximum of the singledish beam, and $\beta$ is the slope of the power law number counts which is required to correct for the Malmquist bias $(\beta \sim-2)$.

With our ALMA survey we can empirically test this relation by checking the positional offsets of the AS2UDS sources relative to their corresponding SCUBA-2 source position. We check for a systematic astrometric offset between the parent SCUBA-2 S2CLS position and the detected ALMA sources (the offset corrections from the increased SCUBA-2 integration time for the Cycle 1 sources, mentioned in $\S 2.2$, were pre-applied) by calculating median offsets in Right Ascension and Declination. We find a significant median offset of -1 .' $^{\prime} 6 \pm 0 .{ }^{\prime \prime} 1$ in R.A. and -0 . $^{\prime} 6 \pm 0$.' 1 in Dec., a result of a rounding error in the assigning of phase centres to ALMA and we subsequently apply these corrections to the ALMA phase centres when calculating the radial separations between SCUBA-2 positions and ALMA source detections. We note that this error in phase centre pointings could result in a modest reduction in the number of ALMA counterparts detected at the very edge of the ALMA primary beam, as a shift of this magnitude results in a region with an area corresponding to $12 \%$ of the primary beam $\sim 8^{\prime \prime}$ from the intended phase centre falling outside the actual primary beam. We estimate a constraint of $\sim 11$ potentially missed galaxies due to this offset, calculated by assuming a rotationally symmetric distribution of galaxies around the phase centres and counting the number of galaxies in our catalogue detected at the very edges of our primary beams.
In Figure 5 we show the radial offsets as a function of the SNR of the parent single-dish source and overlay the predicted uncertainties on the single-dish positions from Ivison et al. (2007). In agreement with the predicted uncertainties, $63 \pm 3 \%$ of the brightest detected sources are within the 1- $\sigma$ uncertainty and $92 \pm 4 \%$ within the $2-\sigma$ uncertainty. However, the actual functional form of the uncertainty in position with signal-to-noise ratio has previously been used to determine the search radius for counterparts (e.g. Biggs et al. 2011) and therefore we look in more detail at how the trend of median offsets varies as a function of signal-tonoise in Figure 5. The median offsets shows a much flatter trend in comparison to the theoretical expectation, with the faintest SCUBA-2 sources $(\mathrm{SNR}<5)$ median offsets lying significantly below the 1- $\sigma$ theoretical prediction of Ivison et al. (2007), whilst the median for the brighter (SNR > 6) sources lies above. This trend suggests that altering the search radius to identify counterparts based on signal-tonoise could result in a failure to correctly identify the correct counterparts in high significance SCUBA-2 detections. This failure to follow the expected behaviourt is likely due to the presence of more than one sub-millimetre galaxy in a map of a single-dish source, which is not accounted for by the model, such 'multiplicity' is particularly prevalent in brighter sources (e.g. Stach et al. 2018).

\subsection{Flux Recovery}

To determine the reliability of our flux measurements we next compare the total flux recovered from all sources detected in an ALMA map against the corresponding SCUBA2 flux. To do this we correct the SCUBA-2 $850 \mu \mathrm{m}$ fluxes to the ALMA wavelength using a $S_{870} / S_{850}=0.95$ fac- 

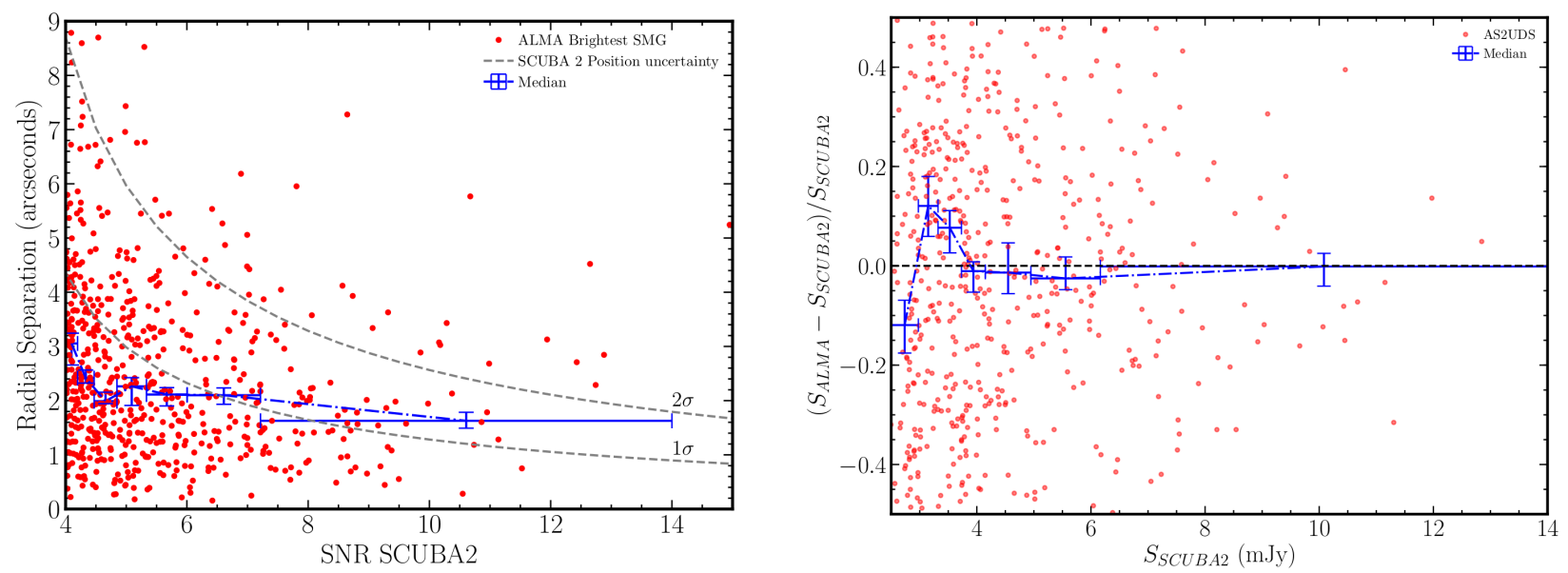

Figure 5. Left: The offsets between the field centres (corrected for the astrometric offsets and the brightest sub-millimetre detected galaxies in the AS2UDS catalogue. As shown by Simpson et al. (2015b) based on our ALMA Cycle 1 pilot programme, these separations are consistent with the predicted uncertainties in source positions for SCUBA-2 detections from Ivison et al. (2007) overlaid as the dashed lines. We also plot the median offsets in bins of equal number of brightest sources which show a mostly flat distribution suggesting a fixed search radius for counterparts as opposed to a SNR dependant one would likely be equally effective, most likely due to the influence of secondary components in the maps. Right: The recovered flux fraction as a function of original SCUBA-2 flux. Each point is the integrated flux for all sources detected in a field within the primary beam centred on the original S2CLS position. Points above zero indicate maps where we recover a greater flux than the original S2CLS detection, a result of either noise boosting or sources being detected near the edge of the map thus are primary beam corrected to higher fluxes, and points below zero are maps where we failed to recover all the flux from the parent single-dish source. Overlaid is the median in bins of equal number of ALMA maps that show that for SCUBA-2 sources above $S_{850} \sim 3.5 \mathrm{mJy}$ we on average recover all the single-dish flux in our ALMA maps.

tor derived from the ALESS survey composite spectral energy distribution (SED) (Swinbank et al. 2014) redshifted to $z=2.5$ (Figure. 5). For SCUBA-2 sources brighter than $S_{850} \geq 4$ mJy beam $^{-1}$ we have a median recovery rate of $97_{-2}^{+1} \%$ of the flux in the ALMA pointings, which is well within the systematic flux uncertainties for both SCUBA2 (5-10\%, Dempsey et al. 2013) and ALMA. Therefore we can be confident that all significant contributors to the sub-millimetre fluxes are successfully recovered in this flux regime. However the binned median flux recovery is $87_{-8}^{+5} \%$ for $S_{850}=2.9-3.1 \mathrm{mJy}$ beam $^{-1}$ and $71 \pm 6 \%$ at $S_{850}=$ 2.5-2.9 $\mathrm{mJy}$ beam $^{-1}$. These medians are biased low by the blank maps with no detected ALMA sources. If we exclude those maps, this results in an $88 \pm 6 \%$ flux recovery at $2.5 \leq S_{850} \leq 2.9 \mathrm{mJy}_{\text {beam }^{-1}}$ and $112 \%$ flux recovery at $2.9 \leq S_{850} \leq 3.1 \mathrm{mJy}$ beam $^{-1}$. Therefore only at the faintest SCUBA-2 fluxes do we see the suggestion of decline in the recovered fraction of the SCUBA-2 flux, and in Section 4.3 we show that for the majority of cases this is likely due to faint sub-millimetre galaxies just below our $4.3 \sigma$ threshold being present in the ALMA maps.

\subsection{Photometric Redshifts}

With the final AS2UDS catalogue matched to the extensive multiwavelength coverage in the UDS field we derive the multiwavelength properties for our SMGs from SED fitting from a maximum of 22 filters $(U, B, V, R, I, z, Y, J, H, K$, IRAC 3.6, 4.5, 5.8, and $8.0 \mu \mathrm{m}$, MIPS $24 \mu \mathrm{m}$, PACS 100 , and $160 \mu \mathrm{m}$, SPIRE 250,350 , and $500 \mu \mathrm{m}, S_{870}$, and $\left.S_{1.4 \mathrm{GHz}}\right)$ using MAGPHYS (Da Cunha et al. 2008). MAGPHYs employs the stellar population synthesis models of Bruzual \& Charlot (2003) with a Chabrier (2003) IMF, combined with a two- component description of the dust attenuation in the ISM and stellar birth clouds Charlot \& Fall (2000) in an energybalance model to ensure consistency between the mid- to farinfrared emission from dust re-processing of the stellar emission and the integrated (dust-attenuated) stellar emission of the galaxy (details in: Da Cunha et al. 2008). da Cunha et al. (2015) extended this method to include the computation of photometric redshifts simultaneously with the constraints on other physical parameters when fitting the SEDs of dusty star-forming galaxies. We use that version of the code in this paper (for a full description of MAGPHYS-photoz and code release see also Battisti et al. in prep). To test the reliability of the predicted photometric redshifts, MAGPHYS was fitted to 14 photometric bands of 7316 spectroscopic sources from the UKIDSS UDS DR11 photometric catalogue. The median relative difference, $\Delta z=\left(z_{\text {spec }}-z_{\text {MAGPHYS }}\right) /\left(1+z_{\text {spec }}\right)$, was found to be -0.0056 with a standard deviation of 0.30 . Similarly, for the 44 AS2UDS SMGs with spectroscopic redshifts we find a median $\Delta z=-0.02$ with a standard deviation of 0.25 . The complete description of our MAGPHYS SED fitting and the resulting multiwavelength properties is described in $\mathrm{U}$. Dudzevičiūtè et al (in prep.).

\section{RESULTS AND DISCUSSION}

In this section we first catalogue the detected sources in the ALMA maps and discuss the trends with redshifts in our sample and the potential causes for maps which lack detected sources. We also study the properties of those in the sample hosting actively accreting super-massive black holes (SMBHs) and the connection of these galaxies to the formation of massive, passive galaxies at high redshifts. 


\subsection{AS2UDS Catalogue}

The complete AS2UDS catalogue identifies 708 submillimetre galaxies brighter than $S_{870}=0.58 \mathrm{mJy}$ from the original 716 SCUBA-2 sub-millimetre sources, roughly five times larger than the Miettinen et al. (2017) study in COSMOS or ALESS (Hodge et al. 2013). In Table 1 we present the AS2UDS catalogue.

From the 716 ALMA maps: one contains four SMG detections, six have three SMG detections, 78 include two, and 530 detect just a single SMG above $4.3 \sigma$. The majority of the maps containing multiple SMGs correspond to the brighter SCUBA-2 sources, so as Stach et al. (2018) showed the rate of occurrence of multiple counterparts is $26 \pm 2 \%$ in SCUBA-2 sources with fluxes brighter than $S_{850} \geq 5 \mathrm{mJy}$ and $44 \pm 16 \%$ at $S_{850} \geq 9 \mathrm{mJy}$. The presence of these multiple strongly star-forming galaxies in close proximity may be hinting at a role for major mergers in driving the enhanced star-formation rates in some of these systems. Indeed, the small subset of AS2UDS covered by the high-resolution HST imaging (see Figure 3) indicates that many of the SMGs are morphologically complex, with close companions and/or structured dust obscuration, consistent with the HST imaging for the ALESS survey (Chen et al. 2015). From visual inspection, independently carried out by two of the authors, the CANDELS coverage suggests $50 \pm 10 \%$ of our SMGs are either clear mergers or are disks with likely companions with similar colours on $<20 \mathrm{kpc}$ scales, and we group these two visual classifications as 'likely interacting'. The median redshifts for the 'likely interacting' SMGs is $z_{\text {phot }}=2.2 \pm 0.1$, significantly lower than the median redshift for the whole SMG sample (see §4.2). Indeed, if we cut our sample to SMGs at redshifts $z_{\text {phot }}<2.75$, the redshift range at which we could reasonably expect to detect interactions in the CANDELS imaging then this 'likely interacting' classification increases to $17 / 21$ or $\sim 80 \%$ of our SMGs, again this is consistent with Chen et al. (2015). This small subset in the CANDELS imaging strongly hints that major mergers are playing a role in driving the enhanced star-formation rates that result in our galaxies being selected in the sub-millimetre maps.

In Figure 6 we show the apparent magnitude/flux density distributions for the AS2UDS SMGs in $V, K, m_{3.6}, S_{870}$, and $1.4 \mathrm{GHz}$ bands and for comparison the corresponding distributions of the ALESS SMGs (Hodge et al. 2013; Simpson et al. 2014). The median ALMA flux density for the AS2UDS SMGs is $S_{870}=3.73_{-0.10}^{+0.03} \mathrm{mJy}$, very similar to the $S_{870}=3.5 \pm 0.3 \mathrm{mJy}$ for ALESS. Of the $708 \mathrm{SMGs}, 529(75 \%)$ have $K$-band counterparts in the UKIDSS-UDS DR11 catalogue to $K \leq 25.9 \mathrm{mag}$. However, excluding ALMA SMGs which fall either outside the DR11 WFCAM $K$-band image or in regions which are flagged as being shallower, the detection rate corresponds to $84 \pm 4 \%(484 / 577)$. The median apparent magnitudes for these AS2UDS SMGs are $V=26.1 \pm 0.1, K=22.8 \pm 0.1, m_{3.6}=21.65_{-0.03}^{+0.06}$. These values are all in good agreement with the equivalent measurements for the ALESS sample of $V=26.1_{-0.1}^{+0.2}, K=23.0_{-0.1}^{+0.3}$, and $m_{3.6}=21.8 \pm 0.2$. Hence we conclude that these two ALMA follow-up surveys of single-dish detections find SMGs with multi-wavelength magnitude distributions in excellent agreement with each other. These photometric properties demonstrate the dusty nature of the target populations with an increasing number of non-detections towards the bluer opti- cal wavebands, e.g. only $64 \pm 2 \%$ of $K$-band detected SMGs are detected in the $V$-band brighter than $V<27.8$. We note that, unlike in the pilot AS2UDS sample (Simpson et al. $2015 \mathrm{~b}$ ), in the full sample there is no evidence that a lack of a $K$-band detection is a function of the $S_{870}$, with a median ALMA flux of $S_{870}=3.65_{-0.08}^{+0.12} \mathrm{mJy}$ for the $K$-detected SMGs and $S_{870}=3.5_{-0.1}^{+0.2} \mathrm{mJy}$ for the $K$-undetected SMGs, the same trend was found in Cowie et al. (2018).

\subsection{Redshift Distribution and Trends}

The photometric redshift distribution for all 708 SMGs is shown in Figure 7 . We derive a median redshift of $z_{\text {phot }}=2.61 \pm 0.09$ and a tail at higher redshifts, with $33_{-2}^{+3} \%$ of galaxies at $z_{\text {phot }}>3$. This median redshift is consistent with the Cycle 1 pilot sample of bright SMGs from Simpson et al. (2015b) of $z_{\text {phot }}=2.65 \pm 0.13$ (or $z_{\text {phot }}=2.9 \pm 0.2$ including optically faint SMGs without precise photometric redshifts). The more direct comparison to our sample are the MAGPHYS-derived redshift distribution for ALESS from da Cunha et al. (2015) who found a median redshift of $z=2.7 \pm 0.1$ with a similar fraction of galaxies in the $z_{\text {phot }}>3$ tail of $38_{-6}^{+7} \%$.

We next compare our photometric redshift distribution with previous spectroscopic redshift surveys selected at $850 \mu \mathrm{m}$ SMGs. Chapman et al. (2005) found a median redshift for their 73 radio-detected SMGs of $z=2.2 \pm 0.1$ ( $z=2.3 \pm 0.2$ after statistically correcting for the radio bias). The lower median redshifts is unsurprising given the lack of a negative $K$-correction at radio wavelengths. We can confirm this by looking at the AS2UDS SMGs with radio bright counterparts where we find a median redshift of $z=2.4_{-0.9}^{+0.3}$, which is in reasonable agreement with the Chapman et al. (2005) sample. The spectroscopic sample of Danielson et al. (2017), which consisted of 52 ALMAconfirmed SMGs detected in the ALESS catalogue found a median redshift of $z=2.40 \pm 0.10$, in rough agreement with the photometric redshift distribution of AS2UDS, however, this spectroscopic redshift sample is biased to optically and near-infrared bright counterparts.

There have also been wide-field single-dish surveys at longer wavelengths, $\sim 1-1.2 \mathrm{~mm}$, which are now being similarly followed up with sub/millimetre interferometers (e.g. Smolčić et al. 2012; Miettinen et al. 2017). In particular, Brisbin et al. (2017) obtained ALMA interferometric observations of $1291.25 \mathrm{~mm}$ sources in COSMOS and determined a median redshift (from a heterogeneous mix of spectroscopic, photometric and colour-based estimates or limits) of $z \sim 2.48 \pm 0.05$ for a sample with an equivalent $870-\mu \mathrm{m}$ flux of $S_{870} \gtrsim 6 \mathrm{mJy}$. This is in reasonable agreement with our median redshift, given the different flux and waveband selections.

We can also compare our median redshift with those derived for the typically fainter samples of SMGs from 'blank field' ALMA surveys (e.g. Walter et al. 2016; Dunlop et al. 2017). The ALMA 'blank field' surveys are usually undertaken at longer wavelengths than our $870-\mu \mathrm{m}$ observations, normally in Band 6 at $\sim 1.1-1.3 \mathrm{~mm}$, to exploit the larger ALMA primary beam to increase the area coverage. Three ALMA surveys of blank fields have reported median redshifts for their samples, although we caution that these all 
Table 1. The AS2UDS Catalogue. The ALMA_ID comprises a 4-digit numbers giving the field identifier from the parent S2CLS survey and a final digit which identifies the individual galaxies detected from each S2CLS source with ascending numbers for descending flux. The source co-ordinates are the source centroids from SEXTRACTOR from the $u v$-tapered detection maps. Field rms is the sigma-clipped rms across the entire map and are plotted in Figure 2. PB Correction gives the primary beam-correction factor at each source location, a higher number indicating galaxies further away from the ALMA phase centre. The S2CLS Flux columns lists the flux-deboosted $850 \mu \mathrm{m}$ flux from Geach et al. (2017). The Aper SNR column is the 0". 5 diameter aperture flux SNR used to select of galaxies in the catalogue and the Final Flux is the 1"’0 diameter aperture flux of the galaxy with the primary beam, aperture, and flux boosting corrections applied. The complete catalogue also contains information on which ALMA cycles the galaxies were observed, the individual flux corrections and the restoring beam properties of the maps.

\begin{tabular}{cccccccc}
\hline ALMA_ID & $\begin{array}{c}\text { Source_RA } \\
\text { J2000 }\end{array}$ & $\begin{array}{c}\text { Source_Dec } \\
\text { J2000 }\end{array}$ & $\begin{array}{c}\text { Field RMS } \\
\left(\text { mJy beam }^{-1}\right)\end{array}$ & PB Correction & $\begin{array}{c}\text { S2CLS Flux } \\
(\mathrm{mJy})\end{array}$ & $\begin{array}{c}\text { Aper SNR } \\
\text { ALMA Final Flux } \\
(\mathrm{mJy})\end{array}$ \\
\hline AS2UDS0001.0 & 34.62777 & -5.52545 & 0.38 & 1.01 & $52.6 \pm 0.0$ & 35.7 & $30.2 \pm 0.86$ \\
$\ldots$ & $\ldots$ & $\ldots$ & $\cdots$ & $\cdots$ & $\cdots$ & $\cdots$ & $\cdots$ \\
\hline
\end{tabular}

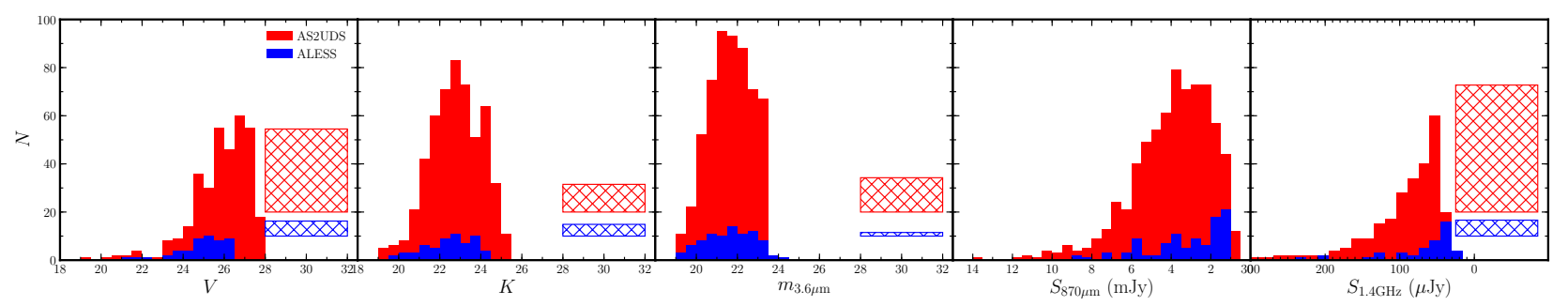

Figure 6. Apparent magnitude distributions in the $V, K$ and $3.6 \mu \mathrm{m}$ wavebands and the flux densities in $870 \mu \mathrm{m}$ and $1.4 \mathrm{GHz}$ for AS2UDS. For comparison we show the same distributions for the earlier ALESS survey, and in both cases the hatched regions showing the non-detections. The median magnitudes/fluxes for AS2UDS are $V=26.1 \pm 0.1, K=22.8 \pm 0.1, m_{3.6}=21.65_{-0.03}^{+0.06}, S_{870}=3.73_{-0.10}^{+0.03} \mathrm{mJy}$, and $S_{1.4 \mathrm{GHz}}=86_{-5}^{+2} \mu \mathrm{Jy}$, with the estimates for the smaller ALESS survey in good agreement. These illustrate that typical SMGs have red optical-near infrared colours and so are very faint in the optical with a large fraction of the population undetected in the bluest wavebands. In contrast, most of the SMGs are detected at $3.6 \mu \mathrm{m}$.

study fields in the GOODS-South area and so are not truly independent. Aravena et al. (2016) report a median redshift of $z=1.7 \pm 0.4$ for a sample of nine galaxies with an $870-\mu \mathrm{m}$ equivalent flux limit of $S_{870} \approx 0.1 \mathrm{mJy}$, Dunlop et al. (2017) report $z=2.0 \pm 0.3$ for 16 sources with optical/near-infrared counterparts and $S_{870} \approx 0.3 \mathrm{mJy}$ and Franco et al. (2018) find $z=2.9 \pm 0.2$ for a sample of 20 galaxies with $S_{870} \gtrsim 2 \mathrm{mJy}$. These studies are challenging and the results from all of them are currently limited by statistics, but there is a hint that the deeper surveys are finding lower median redshifts than from our bright sample, in the same sense as the trend we discuss below.

We next study the variation in median redshift with 870- $\mu \mathrm{m}$ flux density within AS2UDS. It has long been claimed that there is variation in redshift with submillimetre flux, in the sense that the more luminous submillimetre sources typically lie at higher redshifts. This behaviour was first seen in powerful high-redshift radio galaxies Archibald et al. (2001), but the existence of a similar trend has been claimed for sub-millimetre galaxies, although these claims have typically suffered from incomplete, heterogeneous or highly uncertain redshift estimates (e.g. Ivison et al. 2002; Pope et al. 2005; Younger et al. 2007; Smolčić et al. 2012; Koprowski et al. 2014; Brisbin et al. 2017). The initial search for a $z-S_{870}$ trend in the ALESS sample (Simpson et al. 2014) and in the initial bright pilot for AS2UDS (Simpson et al. 2015b) both found weak trends. Moreover, these were based on relatively small and incomplete samples owing to the reliance on optical-near-infrared photometric redshifts. When allowance was made for this incompleteness, the trends were not statistically significant.

One benefit of using MAGPHYS for our photometric redshift analysis is that we can obtain more complete and homogeneous redshifts estimates, not only for those sources detected in the optical to near-infrared bands, but also where only longer wavelength constraints are available. So, we begin by determining if the SMGs which are undetected in the $K$-band are at higher redshift than those which have a $K$-band counterpart, as would be required for their to be a trend of $z-S_{870}$. For galaxies with $K$-band counterparts we find a median $z_{\text {phot }}=2.6 \pm 0.1$ and for the $K$-band nondetections we find a median $z_{\text {phot }}=3.0 \pm 0.1$, suggesting that indeed the population of $K$-faint SMGs, whilst not deviating dramatically in distribution of $S_{870}$ as mentioned above, do represent a higher redshift subset of SMGs.

In Figure 8 we show the photometric redshifts as a function of the ALMA $870 \mu \mathrm{m}$ flux density for AS2UDS using our complete MAGPHYS-derived photometric redshifts. We over plot a linear fit to the median redshifts in bins of equal number of galaxies. This fit shows a trend of median redshift with $870 \mu \mathrm{m}$ flux density with a highly significant gradient of $0.09 \pm 0.02 \mathrm{mJy}^{-1}$. The significance of this result is perhaps surprising given the absence of a strong trend in either ALESS (Simpson et al. 2014) or our Cycle 1 pilot programme (Simpson et al. 2015b). However, the most likely explanation is simply the much smaller sample sizes $(n<100)$ in those studies. To test this we randomly draw samples of 100 galaxies from the complete AS2UDS cata- 


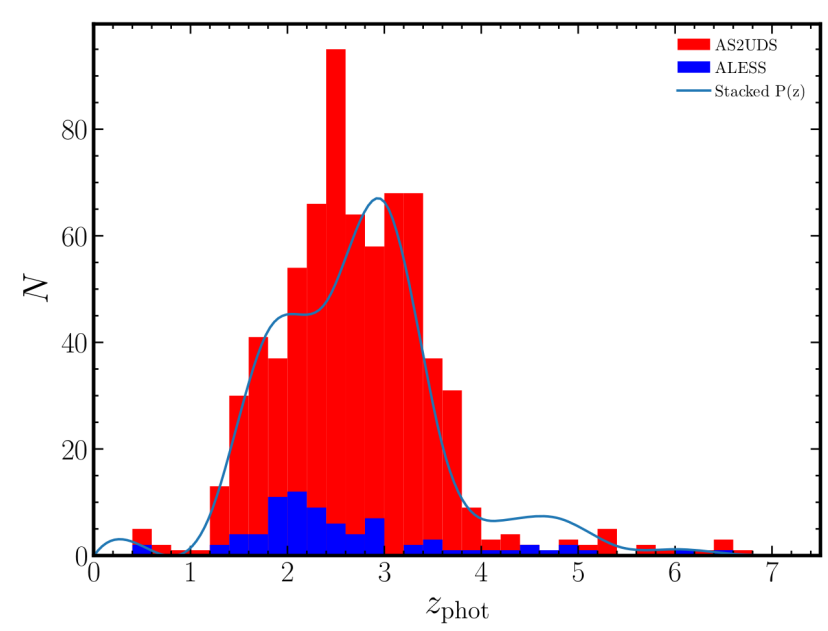

Figure 7. The photometric redshift distribution for the AS2UDS SMGs based on MAGPHYS analysis (Dudzevičiūtè et al. in prep). We determine a median redshift of $z_{\text {phot }}=2.61 \pm 0.09$, consistent with the ALESS photometric redshift distribution (da Cunha et al. 2015), which has a median $z_{\text {phot }}=2.7 \pm 0.1$. The ALESS distributions also display a fraction of sources at $z_{\text {phot }}>3$ galaxies, $38_{-6}^{+7} \%$, that agrees with that derived here for our large AS2UDS sample, $33_{-2}^{+3} \%$. To illustrate the influence of the asymmetric redshift uncertainties we also overlay the stacked probability distributions for the photometric redshifts of all AS2UDS SMGs from MAGPHYS. This closely resembles the median photometric redshift distribution, suggesting that the full combined photometric redshift is not sensitive to any secondary redshift solutions in individual sources.

logue 500 times and repeat the same fitting procedure. In this case we recover an average gradient of $0.08 \pm 0.06 \mathrm{mJy}^{-1}$ confirming that the smaller samples employed in all previous tests mean that it would be impossible to reliably recover the trend we see at higher than $\sim 1.5 \sigma$ significance. In addition, to confirm that our detection incompleteness isn't driving this trend we perform the same trend fitting to the galaxies with fluxes $\mathrm{S}_{870}>4 \mathrm{mJy}$, again finding a similar gradient of $0.08 \pm 0.03 \mathrm{mJy}^{-1}$.

What is the physical process responsible for the $z-S_{870}$ trend we see in AS2UDS? Due to the negative $K$-correction of the dust SEDs in typical SMGs, across the redshift range $(z \sim 1.5-5)$ that we measure this trend, the population have a roughly constant observed flux density at a fixed luminosity (and temperature). Hence the brighter galaxies found at the higher redshifts are intrinsically more luminous. The trend we see then suggests there is a strong luminosity evolution for our SMGs out past $z>3$, with the most far-infrared luminous galaxies being found in greater numbers in the early Universe. This could be symptomatic of galaxy 'downsizing' (Cowie et al. 1996), i.e. the more massive galaxies are forming at higher redshifts. We will return to the issue of potential evidence of 'downsizing' on a forthcoming paper on the clustering of the AS2UDS SMGs in the UDS field (Stach et al. in prep.).

Finally, to attempt to model the variation in median redshifts, we employ the Béthermin et al. (2015) models of SMG number counts and redshift distributions to model the median redshifts of surveys from two variables; the wave-

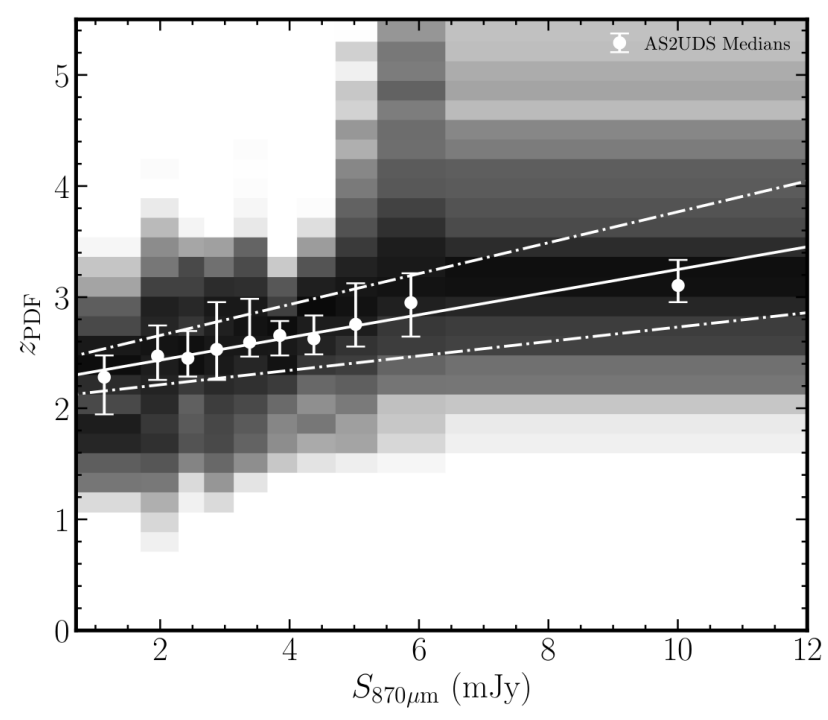

Figure 8. The trend between photometric redshifts and ALMA $870 \mu \mathrm{m}$ flux density for AS2UDS SMGs. We bin the galaxies by ALMA $870 \mu \mathrm{m}$ flux density with bins of equal galaxy numbers and find that the median redshift and flux of each bin. These show a significant trend of increasing redshift with increase flux. The linear fit to this trend has a gradient of $0.09 \pm 0.01 \mathrm{mJy}^{-1}$ and we plot this as the solid line and the $3-\sigma$ errors for this fit as the dashed lines. In addition to the medians of the bins, the greyscale background shows the stacks of the redshift probability density functions for each bin, which also display the same flux density evolution.

length of selection of the SMGs and the flux density depth of the survey. The Béthermin et al. (2015) model suggests that surveys at longer wavelengths will recover a higher median redshift and that, due to the luminosity evolution, the fainter sources found in deeper surveys will predominantly lie at lower redshifts. Whilst this phenomenological model reproduces the broad trend we see in AS2UDS, as well as the comparatively low median redshifts of Aravena et al. (2016) and Dunlop et al. (2017) due to their fainter flux limits, it does under-predict the median redshift for our survey. For a $S_{870}>4$ mJy flux limited sample the model predicts $z \sim 2.6$ compared to our $z=2.8 \pm 0.1$, and this $\Delta z \sim-0.2$ offset between the model and our survey roughly persists across any choice of faint flux limit in our catalogue, indicating that further tuning of this phenomonological model may be needed.

\subsection{Blank Maps}

There are 101 ALMA maps of SCUBA-2 sources in which we find no ALMA detected source above our $4.3 \sigma$ detection threshold, these are termed 'blank' maps. This rate of nondetection is considerably higher than the expected $2 \%$ falsepositive rate predicted from Geach et al. (2017) for the initial SCUBA-2 catalogue. Whilst these 'blank' maps typically correspond to the fainter single-dish sources: the median SCUBA-2 flux of the 'blank' maps is $S_{850}=4.0 \pm 0.1 \mathrm{mJy}$, compared to $S_{850}=4.5 \pm 0.1 \mathrm{mJy}$ for the whole sample, the SCUBA-2 noise properties of the 'blank' maps are consistent with those of the maps with detections. To confirm that 
these are not dominated by false positive detections in the parent SCUBA-2 catalogue we stack the Herschel/SPIRE and SCUBA-2 maps at the locations of the 101 'blank' map sources ranked in four bins of their SCUBA-2 flux and further sub-dividing the faintest quartile in SCUBA-2 flux into two independant halves, shown in Figure. 9.

For each subsample we detect emission in the Herschel/SPIRE at 250 and $350 \mu \mathrm{m}$ and even when further subdiving the faintest subsample into two there is still emission detected at $250 \mu \mathrm{m}$ strongly suggesting these ALMA-blank maps are not just the result of spurious single-dish sources. To compare the $850 \mu \mathrm{m}$ fluxes implied by these SPIRE detections with the original SCUBA-2 measurements, we predict $850 \mu \mathrm{m}$ flux from the SPIRE stacks by fitting a modified blackbody SED with $z=2.5$, a dust temperature $T_{d}=35 \mathrm{~K}$, and emissivity $\beta=1.5$ to each of the $250 / 350 / 500 \mu \mathrm{m}$ stacked fluxes. In Figure 10 we show the predicted $850 \mu \mathrm{m}$ flux from these SED fits against the original detected $850 \mu \mathrm{m}$ emission from SCUBA-2. Whilst there is significant scatter in these estimates, the $850-\mu \mathrm{m}$ fluxes predicted from the extrapolation of the stacked Herschel fluxes is consistent with that observed by SCUBA-2. We view this as a strong confirmation that the majority of these SCUBA-2 sources with ALMA 'blank' maps are not spurious detections in the original S2CLS catalogue.

We conclude that spurious single-dish detections are not the dominant cause for 'blank' maps, therefore, we next examine possibilities for missing ALMA counterparts in our ALMA maps of these sources. An alternative possibility is multiplicity (e.g. Karim et al. 2013), where a single-dish source splits into more than one, fainter galaxies. The combined flux from these galaxies would recover the singledish flux, but individually each galaxy is below our detection threshold. To check if this is a possibility we look for over-densities of candidate $K$-band detected galaxies in our ALMA blank maps in comparison to a 'random' location within the UKIDSS UDS coverage. In Figure 10 we show the MAGPHYS photometric redshift distributions for both the $K$ band detected sources within the primary beams of all 101 'blank' maps and for a 'random' sample covering the same area as the 101 ALMA primary beams, but randomly distributed across the UDS field. We can see an excess of $K$ selected galaxies in the ALMA-blank maps. The majority of this excess arises from galaxies at redshifts of $z \sim 1.5-4$, corresponding to the redshift range where our detected SMG population peaks, with 153 excess galaxies in this redshift range in the 'blank' map regions compared to the 'random' area (a factor of $1.36_{-0.12}^{+0.13}$ increase). This over-density comprises an average excess in a 'blank' map of $1.5 \pm 0.5 \mathrm{~K}$-band sources (within the expected sub-millimetre galaxy redshift range). Stacking the ALMA emission of all of the galaxies at $z=1.5-4$ in these 'blank' maps recovers an average flux of $S_{870}=0.12 \pm 0.02 \mathrm{mJy}$ corresponding to an average flux per map of $S_{870}=0.7 \pm 0.1 \mathrm{mJy}$ to be split between the $\sim 1.5$ excess sub-millimetre-bright galaxies in these regions, suggesting a typical flux of $S_{870} \sim 0.5 \mathrm{mJy}$. This excess of sources and detected sub-millimetre flux is consistent with the interpretation of the 'blank' maps as resulting from the single-dish source comprising flux more than one, faint, submillimetre galaxy below our detection threshold.

To test this, in Cycle 5 we re-observed ten of the brightest S2CLS sources which returned no ALMA detections in our Cycle 3 and 4 maps. To both test for the presence of multiple faint SMGs in these fields and to eliminate the possibility of non-detection due to source flux being resolved out in the interferometric images, these observations were much deeper $\left(\sigma_{870}=0.085 \mathrm{mJy}_{\text {beam }}{ }^{-1}\right)$ and at much lower resolution than the original maps (synthesised beam: $0{ }^{\prime \prime} 81 \times 00^{\prime \prime} 54$ ). From the ten single-dish sources we detect $16>4.3 \sigma$ SMGs. We find four SMGs in the ALMA map of a single SCUBA-2 source which had previously had a 'blank' map, two SMGs in each of a further four maps, four SCUBA-2 sources which have only a single corresponding SMG and just one ALMA 'blank' map which remains blank in these deeper observations (UDS0101, which may be a true false-positive in the S2CLS catalogue). These deeper observations thus confirm that multiplicity is a significant driver of the 'blank' maps and that it remains an issue even for faint SCUBA-2 sources.

The ALMA galaxies in these re-observed 'blank' maps, as expected, are amongst the faintest in the catalogue, with a median deboosted flux of $1.13_{-0.16}^{+0.44} \mathrm{mJy}$. Even at the $\sim 4 \times$ depth of these observations, and with the high detection rate of SMG counterparts, we still only recover an average of $52_{-3}^{+5} \%$ of the S2CLS source flux due to flux boosting. Whilst these SMGs are faint, we do not see any evidence from their beam-deconvolved continuum sizes that their $870-\mu \mathrm{m}$ emission is more spatially extended than the galaxies detected in the higher resolution maps. This suggests that the missing flux, at least in these ten maps, is not a result of flux being resolved out in either our original higher-resolution imaging or the new deeper and lower resolution observations.

\subsection{AGN Fraction}

SMGs have been proposed as the progenitors for the massive spheroid galaxies seen in the local Universe (Lilly et al. 1999; Smail et al. 2004; Simpson et al. 2014). Locally such galaxies exhibit a strong correlation between the mass of their central super-massive black holes (SMBH) and the stellar mass of the host galaxy (Magorrian et al. 1998; Gebhardt et al. 2000; Ferrarese \& Merritt 2000; Gültekin et al. 2009). The existence of this correlation has been used to argue that there is some form of co-evolutionary growth of the SMBH and the surrounding host. This suggestion is supported by observations of the star-formation history and AGN activity of the Universe, which both peak at similar redshifts $z \sim 2$ (Connolly et al. 1997; Merloni 2004; Hopkins et al. 2007; Cucciati et al. 2012; Kulkarni et al. 2018). Support also comes from simulations of galaxy mergers and AGN activity, which predict that galaxy mergers trigger star formation and then the subsequent fueling of the SMBH creates an AGN which quenches the star formation through feedback winds (Hopkins et al. 2008; Narayanan et al. 2010), consistent with the proposed evolutionary path of Sanders et al. (1988). Hence surveying the AGN activity in the SMG population not only provides insights into SMBH growth, but also potentially the evolutionary cycle of SMGs.

\subsubsection{X-ray-selected AGN}

The most reliable method to identify AGNs in galaxies is through the detection of luminous X-ray counterparts. As noted earlier, part of the UDS field has been observed in the 


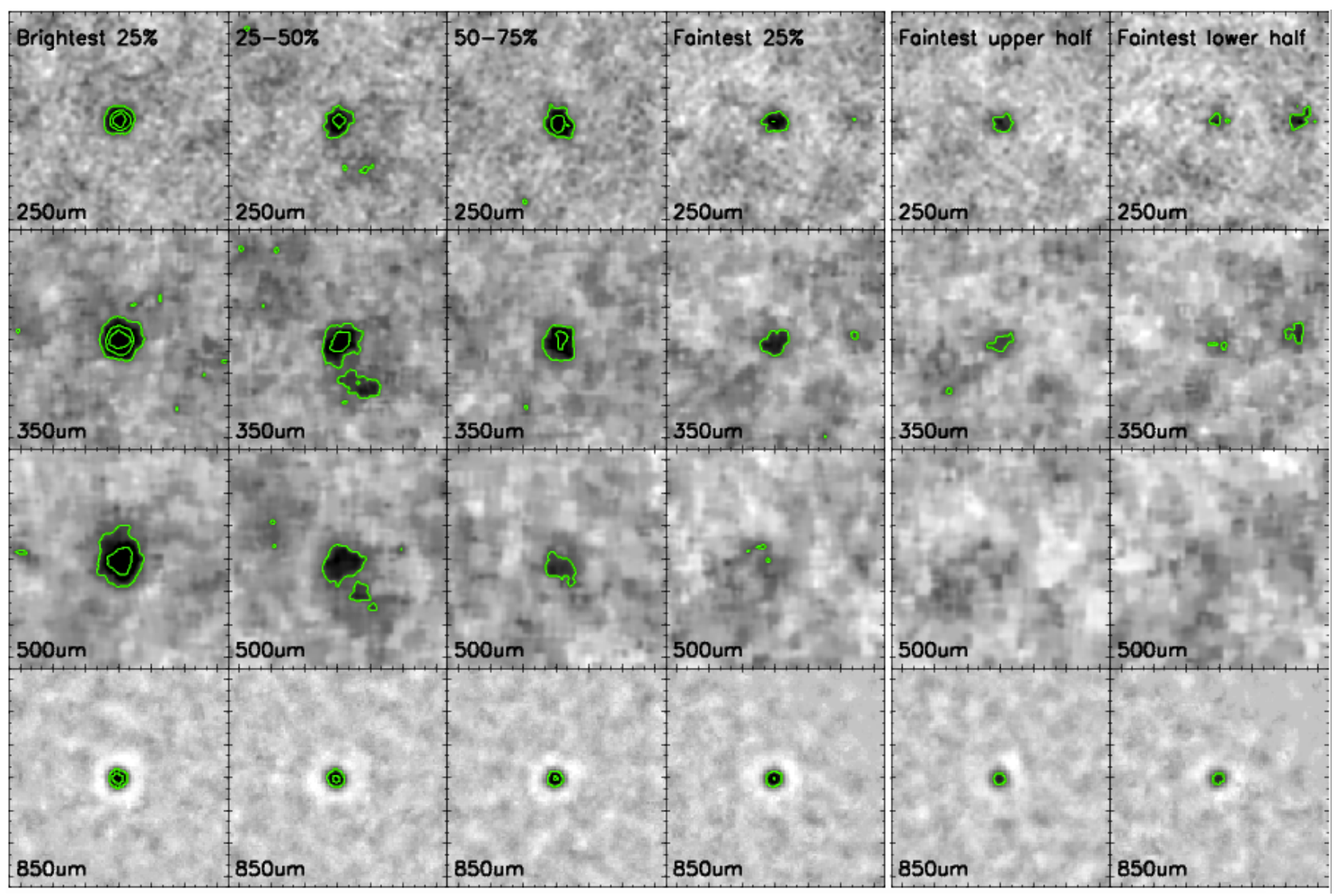

Figure 9. Herschel/SPIRE and SCUBA-2 stacks on the 101 ALMA 'blank' maps in four bins of original SCUBA-2 flux. Even in the faintest SCUBA-2 bin we detect emission at $250 \mu \mathrm{m}$ and $350 \mu \mathrm{m}$. Further splitting the faintest bin into two results in detected emission strongly suggesting these 'blank' maps are not typically the result of spurious single-dish SCUBA-2 detections. Each map is 120 " square and is centred on the SCUBA-2 source position.

X-ray band with Chandra by the X-UDS survey (Kocevski et al. 2018). X-UDS mapped a total area of $0.33 \mathrm{deg}^{2}$, of which the central $\sim 100 \operatorname{arcmin}^{2}$ (coinciding with the CANDELS footprint) is three times deeper than the remainder. A total of 274 SMGs from AS2UDS are covered by the $\mathrm{X}$-UDS observations, with 47 of these lying in the deeper CANDELS region. Considering the high far-infrared luminosities of our SMGs, which is indicative of high starformation rates (which contributes to the X-ray emission), we adopt a conservative full-band X-ray luminosity limit of $L_{\mathrm{X}} \geq 10^{43} \mathrm{erg} \mathrm{s}^{-1}$ for classification as an AGN, consistent with Franco et al. (2018). For consistency, we transform the reported $L_{X}$ from the redshifts quoted in the X-UDS catalogue to those derived from our MAGPHYS analysis, where necessary.

Of the 274 SMGs covered by the X-ray observations, just $21(8 \pm 2 \%)$ are matched to X-ray counterparts in the $\mathrm{X}$-UDS catalogue based on the positional errors quoted for the X-UDS sources. All of these are classed as AGNs with $L_{\mathrm{X}} \geq 10^{43} \mathrm{erg} \mathrm{s}^{-1}$. Of the 47 sources lying within the CANDELS field, only two are matched to X-UDS X-ray sources: AS2UDS0173.0 and AS2UDS0292.0.

The Chandra coverage in the UDS field is relatively shallow for identifying AGN at very high redshifts (c.f. $200-600 \mathrm{ks}$ in UDS, versus $7 \mathrm{Ms}$ in CDF-S). Hence, we also stacked the X-UDS Chandra soft $(0.5-2 \mathrm{keV})$ and hard
(2-8 keV) bands at the positions of the AS2UDS sources which are individually undetected in X-UDS. To perform this stacking we use the CSTACK stacking software ${ }^{2}$. We convert the mean stacked count-rates and uncertainties to fluxes using the conversion factors given in Kocevski et al. (2018), and then to X-ray luminosities by assuming a power-law Xray spectrum with photon index $\Gamma=1.7$. We excluded the 21 SMGs with X-UDS catalogue matches and stacked the remaining 253 SMGs which are individually undetected from the AS2UDS catalogue in three bins of $L_{8-1000 \mu m}$ (derived from MAGPHYS), with the bins chosen to give roughly equal number of sources.

We plot the X-ray and far-infrared luminosities of the 21 individually X-ray detected SMGs and the three composite stacked subsamples in Figure 11. As noted in Alexander et al. (2005a) there appears to be a dividing line between AGN-dominated and starburst-dominated galaxies at $\mathrm{L}_{\mathrm{X}} \sim 0.004 \mathrm{~L}_{\mathrm{FIR}}$. As expected from the Sanders et al. (1988) model of SMG evolution, the SMGs with X-UDS matches cover the entire range of $L_{\mathrm{X}} / L_{\mathrm{FIR}}$ from nearly starformation dominated to the region associated with the AGNdominated quasars. Nevertheless, the majority of our in-

2 Developed by T. Miyaji. The UDS implementation of CSTACK will be made public a year after the publication of Kocevski et al. (2018) 

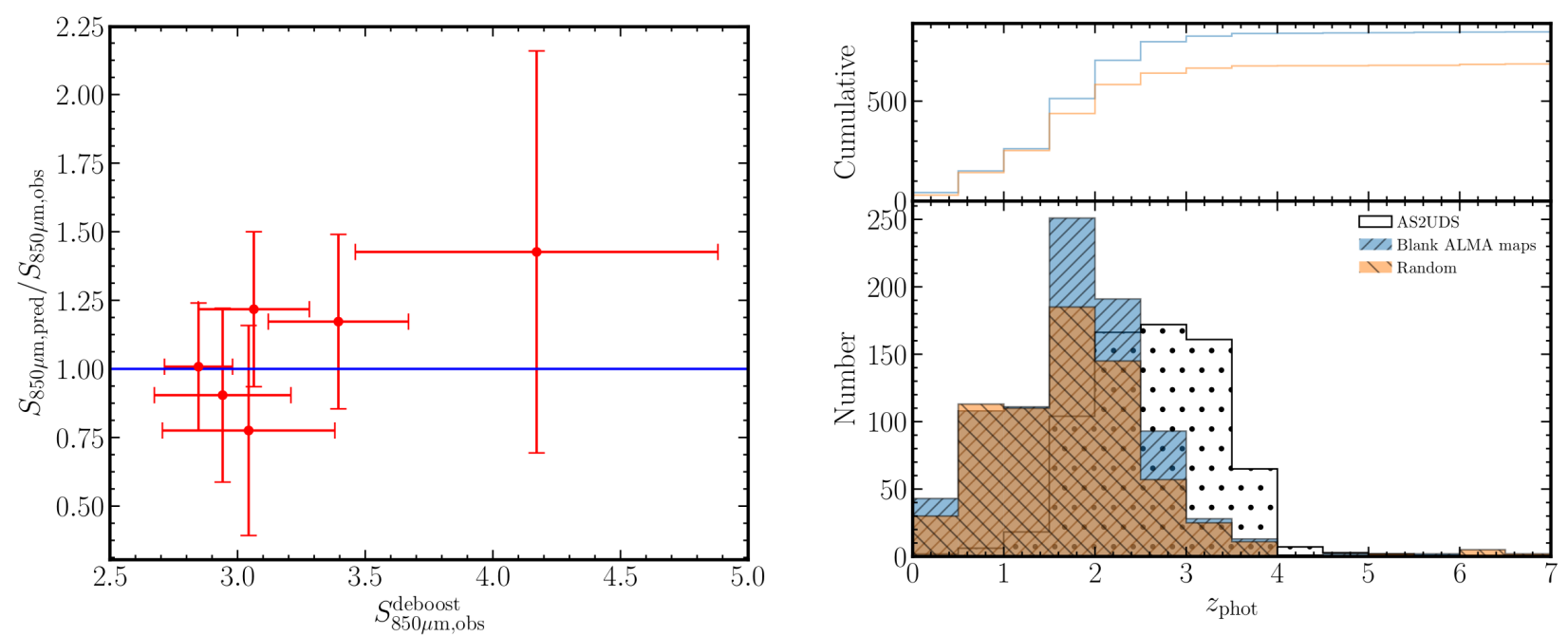

Figure 10. Left: Predicted $850 \mu \mathrm{m}$ flux from fitting SEDs to the stacked Herschel/SPIRE fluxes of sub-samples of SCUBA-2 sources, ranked in terms of SCUBA-2 $850 \mu \mathrm{m}$ flux, where our ALMA observations detected no SMGs. These are compared to the observed SCUBA-2 $850 \mu \mathrm{m}$ flux for the equivalent sub-sample. On average we recover the predicted SCUBA-2 fluxes from the SED fitting which strongly suggests that the majority of these SCUBA-2 sources are real, rather than spurious detections in the parent catalogue. Right: The MAGPHYS photometric redshift distribution of the $K$-band DR11 UKIDSS UDS sources within the primary beam of the ALMA 'blank' maps. This is compared to an identical sized area randomly distributed across the UDS field ('random'). We see a significant excess of galaxies in the 'blank' map regions compared to a random field, corresponding to an excess of 153 galaxies at $z_{\text {phot }}=1.5-4$. This suggests that these apparently 'blank' maps might each contain 1-2 faint SMGs lying at redshifts similar to the distribution of the brighter, detected, SMGs which is also shown.

dividually X-ray-detected SMG show $L_{\mathrm{FIR}} / L_{\mathrm{X}}$ ratios consistent with AGN dominated systems, as expected from their median $L_{\mathrm{X}} \sim 10^{44} \mathrm{erg} \mathrm{s}^{-1}$. However, the three stacked subsamples of individually X-ray-undetected SMGs all exhibit ratios of $L_{X}=(5 \pm 2) \times 10^{-5} L_{F I R}$, more consistent with starburst-dominated systems. This indicates that on-average the X-ray-undetected SMGs are likely to be star-formation dominated and hence the bulk of the SMG population is unlikely to host luminous AGN.

We estimate the black hole mass accretion rates $\left(\dot{M}_{\mathrm{acc}}^{\mathrm{BH}}\right)$ for our SMGs by assuming a $L_{\mathrm{X}}$ to $L_{\mathrm{bol}}$ bolometric correction factor of 15 (Lusso et al. 2012), an efficiency factor $(\epsilon)$ of 0.1 and using Equation 2

$L_{\mathrm{bol}}=\epsilon \dot{M}_{\mathrm{acc}}^{\mathrm{BH}} c^{2}$.

For our X-ray detected SMGs which we classify as 'AGN dominated' this results in $\dot{M}_{\mathrm{acc}}^{\mathrm{BH}}$ in the range of 0.1-1.4 $M_{\odot} \mathrm{yr}^{-1}$. For galaxies to evolve along the local relation of spheroid mass-black hole mass, then the ratio of this accretion rate to the star-formation rate must be $\sim 2 \times 10^{-3}$ (Magorrian et al. 1998; Drouart et al. 2014). Taking the MAGPHYS derived star-formation rates, for our AGN dominated SMGs we find a median $\dot{M}_{\text {acc }}^{\mathrm{BH}} / \mathrm{SFR}$ ratio of $(1.2 \pm 0.5) \times 10^{-3}$. This is slightly below the expected ratio, which may indicate that these AGN-hosting, but strongly star-forming, SMGs are in a phase where their starformation rate is exceeding the corresponding $\mathrm{SMBH}$ mass growth rate. However, the difference is not statistically significant and moreover, there are significant uncertainties; if the efficiency factor was 0.06 (within the acceptable range of $\epsilon$ e.g. Davis \& Laor 2011) then these systems would com- fortably lie on the co-evolutionary track of stellar and black hole mass.

More critically, that is for the relative SMBH and stellar mass growth in the minority of SMGs which show AGN signatures. For the bulk of star-formation-dominated SMGs (which lie within the X-UDS coverage) we find $\dot{M}_{\mathrm{acc}}^{\mathrm{BH}} / \mathrm{SFR}$ ratios of only $\sim 0.1 \times 10^{-5}$ which is significantly lower than that required for co-evolutionary growth to the local relation. Adopting physically plausible values for the bolometric correction or efficiency factor cannot change this conclusion. Therefore, for the bulk of our SMGs to ultimately follow the local relation in spheroid-black hole mass then there must be some subsequent (-or prior) phase of significant growth of the SMBH with comparatively little star-formation, e.g. high redshift quasars with their SMBH accretion rates of $\sim 1-100 M_{\odot} \mathrm{yr}^{-1}$ (Hao et al. 2008).

\subsubsection{Colour-selected AGN}

In addition to our X-UDS matching and stacking we also supplement our search for AGN activity in the SMG sample by employing IRAC colour-colour selections (e.g. Donley et al. 2012). These use the IRAC bands to identify galaxies with strong power-law emission in the restframe nearinfrared, particularly beyond $2 \mu \mathrm{m}$, which is a good indication of the presence of an AGN, even if it is dust obscured.

Across the whole of the UDS there are 383/708 SMGs with coverage in all four IRAC channels (and with photometry that does not suffer from significant contamination from neighbours, see: $\S 2.3$ ), necessary to apply the colour selection from Donley et al. (2012). We show the distribution of these galaxies in Figure 12, but first check that the heavily dustobscured nature of these sources won't lead to them being 


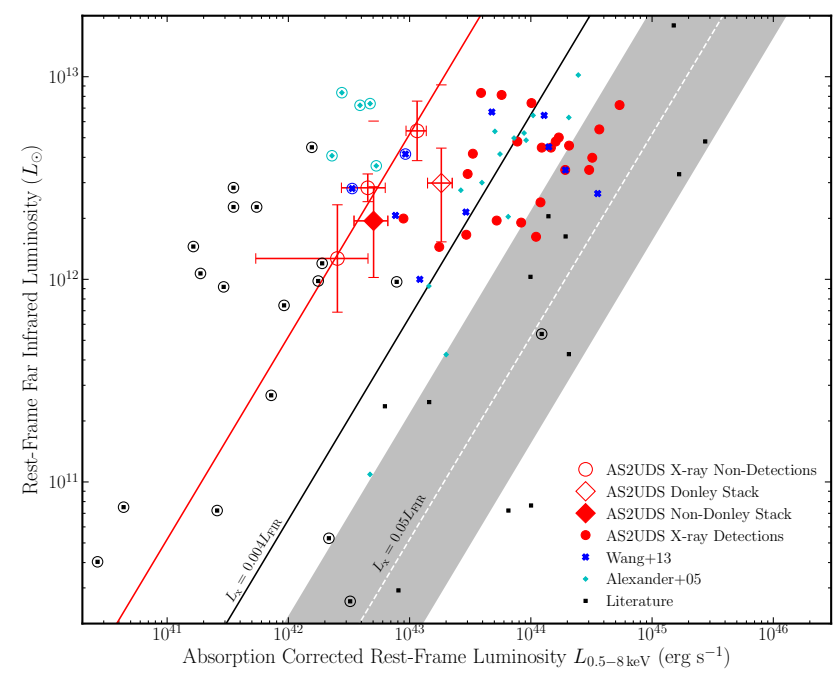

Figure 11. We show the relationship between restframe farinfrared $(8-1000 \mu \mathrm{m})$ luminosity for the X-ray detected AS2UDS SMGs versus their rest-frame $0.5-8.0 \mathrm{keV}$ absorption-corrected luminosities. The 21 galaxies with X-UDS matches from our catalogue (red points) and lie at $L_{\mathrm{x}} \gtrsim 0.004 \mathrm{~L}_{\mathrm{FIR}}$, the approximate dividing line between starburst and AGN dominated galaxies. We have derived mean X-ray fluxes by stacking, using CSTACK, three sub-samples of individually-X-ray-undetected SMGs - where the sub-samples are ranked on $L_{\text {FIR }}$ - these are plotted as open symbols with error bars. These three samples lie below the line dividing starburst and AGN dominated emission. We conclude that the vast majority of the SMG population, $\gtrsim 90 \%$, do not host luminous AGN. Galaxies from the literature are plotted as blue crosses (Wang et al. 2013), cyan diamonds (Alexander et al. 2005b), and black squares for galaxies from the literature compiled by Alexander et al. (2005b). Starburst dominated galaxies are denoted by a circle. The white dashed line and grey shaded region are the median luminosity ratio and standard deviation for the quasars from Elvis et al. (1994). The filled diamond is the results of the stacking of the galaxies outside of the Donley et al. (2012) selection which show, on average, they are star-formation dominated galaxies whereas the the IRAC selected AGNs in the unfilled diamond appear to be, on average, close to the dividing line between star-formation and AGN dominated.

misclassified in this colour-colour space. We therefore plot on Figure 12 the track of the colours expected from the composite SED for ALESS SMGs from Swinbank et al. (2014). This shows that at the highest redshifts, $z>3$ the typical colours of SMGs will mimic that of an AGN, while at lower redshifts the colours of these obscured and actively star-forming galaxies will fall outside the region populated by AGN. For this reason we apply the additional selection cut from Donley et al. (2012) to AS2UDS SMGs in independent redshift slices and we also identify the star-formation dominated highredshift SMGs which display IRAC colours consistent with a redshifted $1.6 \mu \mathrm{m}$ stellar 'bump' and 8.0/3.6 $\mu \mathrm{m}$ flux ratios consistent with the local (Ultra)-luminous infrared galaxies SED templates of Rieke et al. (2009).

We test the efficacy of the Donley et al. (2012) selection by employing CSTACK to derive the average X-ray flux of the 25 SMGs with $z_{\text {phot }}<3$ that are within both the IRAC AGN selection and X-UDS coverage and are not close to another bright X-ray source, and a control sample of 131
SMGs outside of the colour selection region. We show these in Figure 11 and see that the 131 colour-selected SMGs lying outside the Donley et al. (2012) selection do indeed have a $L_{\mathrm{X}} / L_{\mathrm{FIR}}$ ratio consistent with those expected from star formation. However, the stacked X-ray properties of the 25 SMGs at $z<3$ which fall within the Donley et al. (2012) selection provide a more ambiguous $L_{\mathrm{X}} / L_{\mathrm{FIR}}$ ratio, with these sources lying below the threshold to be classified as an AGN. For this reason we choose to view the IRAC-colour selected samples, even at $z<3$, as providing an upper limit on the potential AGN fraction in SMGs.

\subsubsection{AGN fraction in SMGs}

From the SMGs lying within the X-UDS coverage we derive a lower limit on the X-ray detected AGN fraction of 21/274 $(8 \pm 2 \%)$. To estimate an upper limits on the AGN fraction we include the IRAC-colour-selected AGN within this region but employ a $z<3$ cut off for reasons discussed above. With the IRAC-selected AGN and the redshift cut we estimate an upper limit of $45 / 162(28 \pm 4 \%)$ AGN in the AS2UDS population.

The range of potential AGN fraction for our flux-limited sample lies in near the middle of results from earlier work in the literature, e.g. $38_{-10}^{+12} \%$ in Alexander et al. (2005b), (20$29) \pm 7 \%$ in Laird et al. (2010), $18 \pm 7 \%$ in Georgantopoulos et al. (2011) and $\sim 28 \%$ in Johnson et al. (2013), as well as the ALMA-based estimate from ALESS: $17_{-6}^{+16 \%}$ in Wang et al. (2013).

Recently, working with a 1.1-mm selected ALMA sample of SMGs in the GOODS-South field, Franco et al. (2018) reported a high AGN fraction, $\sim 40 \pm 14 \%$. Their SMG sample has an $870-\mu \mathrm{m}$ equivalent flux range of $S_{870} \sim 0.8-$ 3.9 mJy. To match to this Franco et al. (2018) selection, we also estimate the AGN fraction for our fainter $S_{870}<4.0 \mathrm{mJy}$ SMGs and combine the number of our IRAC-selected candidates at $z<3$ with the confirmed X-ray bright SMGs from the X-UDS matching to find an upper limit on the AGN fraction of $26 \pm 5 \%(28 / 109)$ within the X-UDS coverage, consistent with our whole sample. This is lower than the estimate from GOODS-South, but given the significant uncertainty on the latter, we do not give too much weight to this disagreement.

\subsection{Passive Galaxy Progenitors}

SMGs, with their extreme star-formation rates and implied high molecular gas content, could form significant stellar masses $\left(M_{*}\right)$ of $10^{10}-10^{11} \mathrm{M}_{\odot}$ on a timescale of just $\sim 100$ Myrs. This rapid formation of a massive system at high redshifts has led to them being proposed as the progenitors of high-redshift compact quiescent galaxies (Simpson et al. 2014; Toft et al. 2014; Ikarashi et al. 2015), which subsequently evolve into local spheroidal galaxies. Observational support for this evolutionary relation has been claimed from comparisons of the stellar masses of SMGs (Swinbank et al. 2006; Hainline et al. 2011; Toft et al. 2014), the spatial clustering of relative to that of local ellipticals (e.g. Hickox et al. 2012; Chen et al. 2016; Wilkinson et al. 2017) and the compact rest-frame far-infrared sizes of SMGs (Simpson et al. 2017; Hodge et al. 2016), as well as from theoretical mod- 


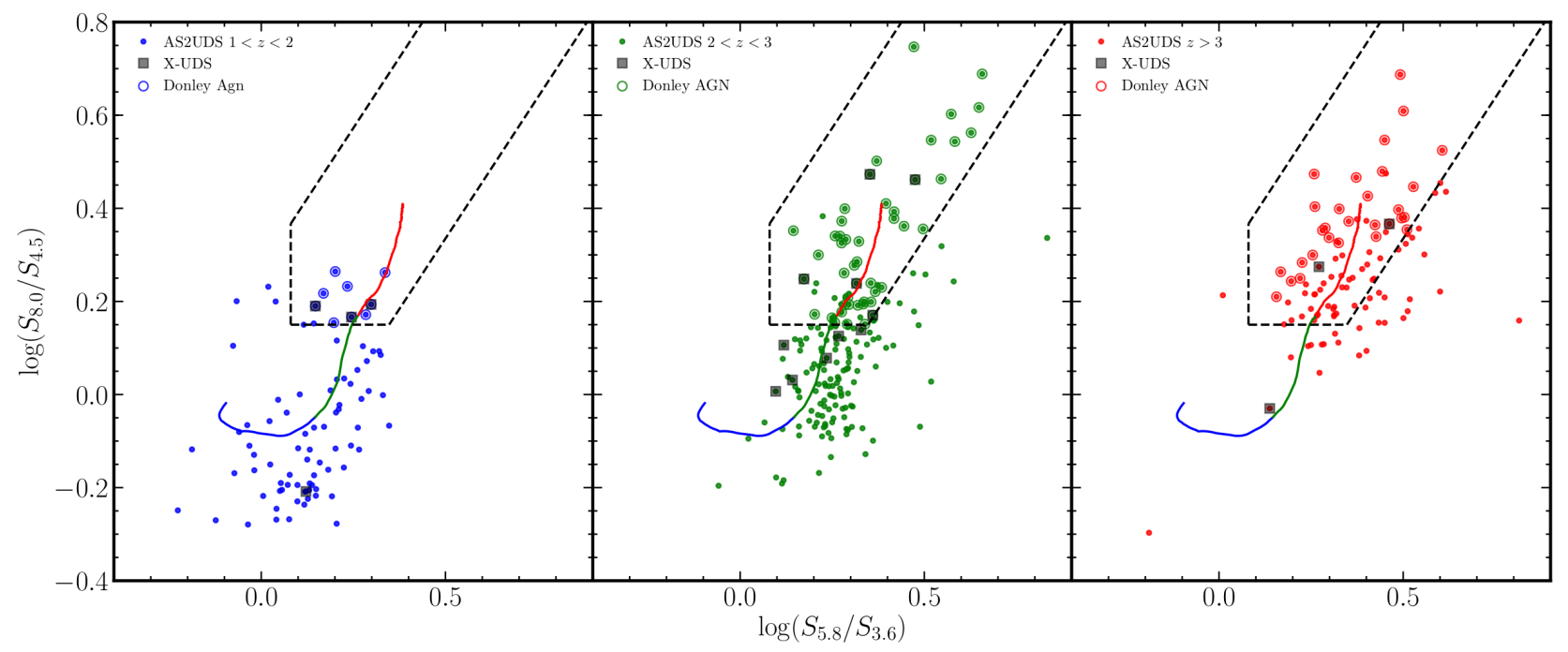

Figure 12. We use the IRAC colour-colour selection described in Donley et al. (2012) to select candidate AGN from the AS2UDS SMG sample, shown here in three redshift slices. We overlay the predicted colours for the ALESS composite SED (Swinbank et al. 2014) as a function of redshift, colour coded in blue for $z=1-2$, green for $z=2-3$, and red for $z>3$. The track illustrates how above $z \gtrsim 3$ the AGN colour selection will potentially be contaminated by star-forming galaxies. The final selection of AGNs based on the 'wedge' and the star-formation dominated cut are circled in their respective redshift colours. In total the IRAC-colour selection finds 125 potential AGN candidates across the entire AS2UDS coverage. However, the most reliable estimate of the AGN fraction comes from the sample lying within the X-UDS region and with redshifts of $z<3$, which yields 37 candidates. The 21 X-UDS X-ray-detected SMGs hosting AGN are marked as black squares.

elling of SMGs and their descendants (González et al. 2011; McAlpine et al. 2019).

Using our large sample with complete redshift information we can test these claimed connections, especially in light of recent advances in the studies of high-redshift passive galaxies. For example Estrada-Carpenter et al. (2019), using HST grism spectroscopy, have constrained the formation redshift and metallicities for a sample of 32 spectroscopically-classified quiescent galaxies at $z=1-1.8$, which are believed to be massive, $\mathrm{M}_{*}>10^{10} \mathrm{M}_{\odot}$. They find that, nearly independent of the observed redshift of the quiescent galaxies, their formation redshift (the epoch where $\gtrsim 70 \%$ of the stellar mass has already formed) is $z_{\text {form }}>2-$ 3 , with a third of their sample having $z_{\text {form }}>3$. Constraints on their metallicities suggesting that these star-forming progenitors must have already enriched to approximately Solar metallicities (which is consistent with the high dust masses of the SMGs as well as the crude estimates of their metallicities from Swinbank et al. (2004); Takata et al. (2006)). Morishita et al. (2018), likewise, looked at the mass accumulation and metallicity history for a sample of 24 apparently massive galaxies $\left(M_{*}>10^{11} \mathrm{M}_{\odot}\right)$ at $z=1.6-2.5$ via SED modelling and inferred that the majority of their sample had formed $>50 \%$ of their mass around $\sim 1.5$ Gyr prior to their observed redshifts, yielding formation ages similar to Estrada-Carpenter et al. (2019).

In Figure 13 we plot the age distribution for massive AS2UDS SMGs and the combined Estrada-Carpenter et al. (2019) and Morishita et al. (2018) formation redshifts for their samples of quiescent galaxies. We adopt a mass limit of $\mathrm{M}_{*}>10^{9.85} \mathrm{M}_{\odot}$, from our MAGPHYS estimated stellar masses to match the passive galaxy samples, this accounts for some continuing star-formation activity and associated stellar mass growth in these systems, although our conclu- sions are not sensitive to this assumption. For a more accurate comparison of 'formation' ages we derive the mean offset from the observed redshift of our SMGs to the 'age' of their mass-weighted stellar population. This offset is calculated from the inferred gas masses, star-formation rates, and stellar masses from MAGPHYS for the AS2UDS sample. We estimate (and apply) a typical $+200 \mathrm{Myr}$ offset to our observed redshifts to correct to a mass-weighted stellar population age which is more representative of the period in which the galaxy was forming the majority of its stellar mass.

Figure 13 shows that the AS2UDS age distribution is comparable to the formation ages which are inferred for massive, quiescent galaxies at $z \sim 1-2.5$ : both distributions peak at lookback times of 11.5-12.5 Gyr, median ages for the distributions are in agreement with $11.4_{-0.2}^{+0.1}$ Gyr for AS2UDS and $11.5 \pm 0.3 \mathrm{Gyr}$ for the comparison sample, and both populations show a younger stellar population $<11$ Gyr tail containing $\sim 30 \%$ of their respective distributions. This provides further support for the claims that the SMG and highredshift massive quiescent galaxy populations may have an evolutionary link.

We can also ask if the number density of the SMGs and high-redshift passive galaxies are consistent with any likely evolutionary cycle. We can derive a co-moving number densities for the subset of the quiescent population from EstradaCarpenter et al. (2019) with formation redshifts $z_{\text {form }}=2-$ 3 (the population where number densities are available), which is $n_{\mathrm{qg}} \sim 2.5 \times 10^{-4} \mathrm{Mpc}^{-3}$. This compares to a number density of $n_{\mathrm{SMG}}=\left(2.8_{-0.1}^{+0.2}\right) \times 10^{-5} \mathrm{Mpc}^{-3}$ for the SMGs in the same redshift range after applying the $M_{*}>10^{9.85} \mathrm{M}_{\odot}$ mass cut. The SMG number density is $11_{-2}^{+3} \%$ of the quiescent galaxy number density estimated by Estrada-Carpenter et al. (2019). However, we also need to correct the apparent 


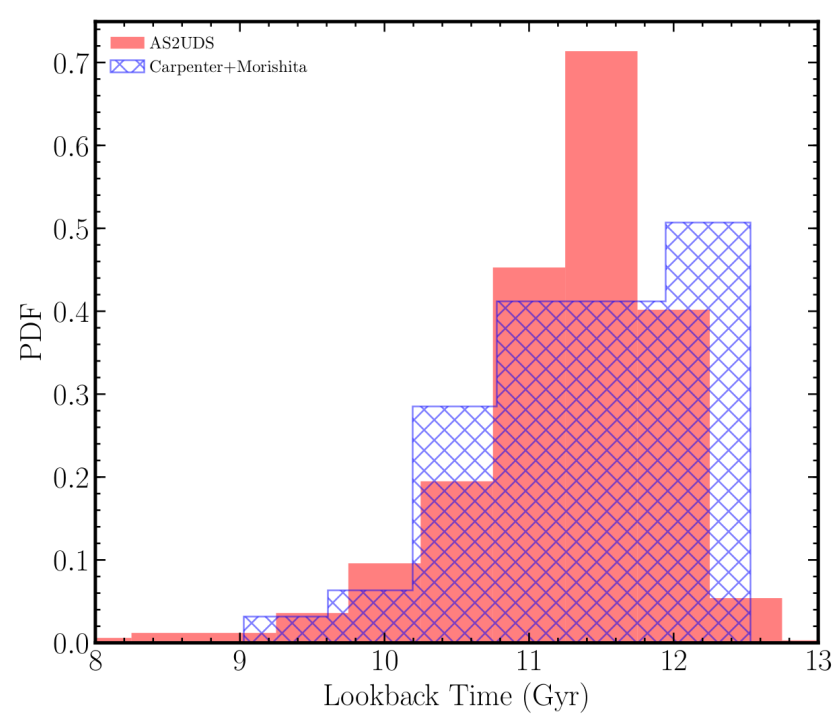

Figure 13. The inferred mass-weighted ages of the AS2UDS SMGs compared to the formation redshift for $z \sim 1-2.5$ passive galaxies from Estrada-Carpenter et al. (2019) and Morishita et al. (2018). The high-redshift quiescent galaxy population across this broad redshift range are found to have similar formation redshifts, which in turn broadly match the redshift distributions for the formation of the SMG galaxy population. This is consistent with the interpretation of the SMGs as likely progenitors for the spectroscopically confirmed quiescent galaxies at $z \sim 1-2.5$.

SMG number density for a duty cycle as they are thought to attain a high star formation rate for a comparatively short duration, compared to the redshift range being considered.

The lifetime of the high-SFR phase of SMGs is dependant on either a simple gas depletion timescale, or through some star formation quenching mechanism e.g. active galactic nuclei feedback. Previous estimates of the lifetimes of the SMG-phase based on gas depletion timescales or clustering analysis have suggested durations of $\sim 40-200 \mathrm{Myr}$ (Tacconi et al. 2006; Swinbank et al. 2006; Riechers et al. 2011; Hickox et al. 2012; Bothwell et al. 2013). We can estimate this average duty cycle duration using a simple model of the SMG evolution by making the following assumptions: (i) the $z=2-3, M_{*}>10^{9.85} M_{\odot}$ AS2UDS SMGs are progenitors of the $z=1-1.8$ quiescent galaxies and likewise all $z=1-1.8$ quiescent galaxies are the descendants of these SMGs, (ii) each SMG has a single burst of intense star-formation. With these assumptions the burst duration can be estimated by

$t_{\text {burst }}=t_{\mathrm{obs}} \times\left(\frac{n_{\mathrm{SMG}}}{n_{\mathrm{qg}}}\right)$,

where $t_{\text {burst }}$ is the burst duration, $t_{\mathrm{obs}}$ is the duration of the epoch we calculated the SMG comoving density $(z=2-$ 3 ), and $n_{\mathrm{SMG}} / n_{\mathrm{qg}}$ are the co-moving number densities for the SMGs and quiescent galaxies respectively. For our measured number density we find a burst duration of $190_{-40}^{+50} \mathrm{Myr}$ which is consistent with the other estimates derived from observed gas masses and star-formation rates of SMGs within this redshift range in the literature (Hickox et al. 2012; Bothwell et al. 2013). Therefore the space-density of dusty star-forming galaxies at $z=2-3$ that form the bulk of the AS2UDS sample, are consistent with that required for them to comprise the progenitors of quiescent galaxy population seen at $z=1-1.8$.

\section{CONCLUSIONS}

We have presented the catalogue for the largest homogeneously-selected sample of sub-millimetre galaxies to date, an ALMA $870 \mu \mathrm{m}$ continuum follow-up survey of $716,>4 \sigma$ single-dish sub-millimetre sources selected from the SCUBA-2 Cosmology Legacy Survey 850- $\mu$ m map of the UKIDSS UDS field. Our deep, high-resolution ALMA observations identified $708>4.3 \sigma$ sources which account for the majority of the flux detected in the parent SCUBA-2 map. The main conclusions of this study are:

- Utilising the extensive multi-wavelength coverage of the UDS field we fit SEDs for our galaxies using MAGPHYS and from these fits derived a median photometric redshift for our galaxies of $z_{\text {phot }}=2.61 \pm 0.09$ with a high-redshift tail comprising $33_{-2}^{+3} \%$ of SMGs with $z_{\text {phot }}>3$.

- From the subset of SMGs with CANDELS imaging we find that $50 \pm 10 \%$ show either clear merger morphologies or have likely companions, displaying similar colours, on < $20 \mathrm{kpc}$ scales. These likely interacting systems have a median redshift at $z_{\text {phot }}=2.2 \pm 0.1$, which is significantly lower than the median photometric redshift. When we select SMGs with redshifts $z_{\text {phot }}<2.75$, to account for the redshift at which we reasonably expect to reliable detect interactions in the CANDELS imaging, then this 'likely interacting' fraction accounts for $\sim 80 \%$ of SMGs. This suggests that the elevated star-formation rates in these systems are driven by mergers.

- With our large sample size we see convincing evidence for evolution in the $S_{870}$ flux density of sources with redshift with a best fit trend gradient of $0.09 \pm 0.02 \mathrm{mJy}^{-1}$. This evolution was not robustly identified in previous smaller surveys due to their limited statistics and we show how reducing our sample size down to $\sim 100$ galaxies results in a statistically insignificant result. The consequence of this trend is that on average our most luminous galaxies are found at higher redshifts in comparison to less active galaxies, a strong indication of galaxy downsizing.

- Through stacking Herschel observations at the positions of the 101 SCUBA-2 sources for our ALMA maps produced no $>4.3-\sigma$ detections, we show that these sources are not dominated by false-positive detections in the parent S2CLS catalogue. We find an overdensity of, on average, $\sim 1.5 \mathrm{~K}$-band sources at the locations of these 'blank' ALMA maps at redshifts $z=1.5-4$ which, combined with the strong evidence from SPIRE-stacking that the original SCUBA-2 flux is real, suggests that the lack of ALMA counterparts is a result of blending of the sub-millimetre emission from $\gtrsim 1-2$ faint galaxies at these positions. We confirm that this is the case with deeper repeat Cycle 5 observations of ten examples of these 'blank' maps which yield 16 new ALMA detections below our previous flux limit. This has significant consequences for the faint-end number counts of sub-millimetre galaxies.

- We identify AGNs associated with our SMG sample by both matching our catalogue to the X-UDS Chandra Xray coverage of the field and also by applying an IRAC colour-colour selection. We estimate a lower limit on our 
AGN fraction from the X-ray detections of $8 \pm 2 \%$ and an upper limit by including our IRAC-colour selected AGNs of $28 \pm 4 \%$. This range is consistent with previous results, although somewhat lower than the most recent results reported for a small sample in the GOODS-S field. We conclude that most sub-millimetre bright galaxies do not host an unobscured or moderately obscured luminous AGN.

- Looking to the most likely candidate descendants for our SMGs we compare the constraints on their redshift and number density (as well as stellar mass and metallicity) to those expected for the progenitors of $z=1-2.5$ quiescent galaxies predicted by Estrada-Carpenter et al. (2019) and Morishita et al. (2018). We find that the properties of the AS2UDS SMG population are consistent with these constraints, with median mass-weighted ages for the SMGs of $11.4_{-0.2}^{+0.1} \mathrm{Gyr}$, in good agreement with the median formation ages for the quiescent galaxies of $11.5 \pm 0.3 \mathrm{Gyr}$. The number density of the SMGs and Estrada-Carpenter et al. (2019) populations are also consistent with this evolutionary link if the typical star-formation burst duration of the SMGs is $\sim 190_{-40}^{+50} \mathrm{Myr}$, which is similar to previous independent estimates.

\section{ACKNOWLEDGEMENTS}

SMS acknowledges the support of STFC studentship (ST/N50404X/1). AMS and IRS acknowledge financial support from an STFC grant (ST/P000541/1). IRS EAC and BG also acknowledge support from the ERC Advanced Investigator program DUSTYGAL (321334). JLW acknowledges support from an STFC Ernest Rutherford Fellowship (ST/P004784/1 and ST/P004784/2). JEG acknowledges support from a Royal Society University Research Fellowship. MJM acknowledges the support of the National Science Centre, Poland through the POLONEZ grant 2015/19/P/ST9/04010; this project has received funding from the European Union's Horizon 2020 research and innovation programme under the Marie Skłodowska-Curie grant agreement No. 665778. T. Miyaji and the development of CSTACK is supported by UNAMDGAPA IN104216,IN111319 and CONACyT 252531. This work makes use of CSTACK (http://cstack.ucsd.edu/ or http://lambic.astrosen.unam.mx/cstack/) developed by Takamitsu Miyaji. The ALMA data used in this paper were obtained under programs ADS/JAO.ALMA\#2012.1.00090.S, \#2015.1.01528.S and \#2016.1.00434.S. ALMA is a partnership of ESO (representing its member states), NSF (USA) and NINS (Japan), together with NRC (Canada) and NSC and ASIAA (Taiwan), in cooperation with the Republic of Chile. The Joint ALMA Observatory is operated by ESO, AUI/NRAO, and NAOJ. This paper used data from project MJLSC02 on the James Clerk Maxwell Telescope, which is operated by the East Asian Observatory on behalf of The National Astronomical Observatory of Japan, Academia Sinica Institute of Astronomy and Astrophysics, the Korea Astronomy and Space Science Institute, the National Astronomical Observatories of China and the Chinese Academy of Sciences (Grant No. XDB09000000), with additional funding support from the Science and Technology Facilities Council of the United Kingdom and participating universities in the United Kingdom and Canada. UKIDSS-DR11 photometry made use of UKIRT. UKIRT is owned by the University of Hawaii (UH) and operated by the UH Institute for Astronomy; operations are enabled through the cooperation of the East Asian Observatory. When (some of) the data reported here were acquired, UKIRT was supported by NASA and operated under an agreement among the University of Hawaii, the University of Arizona, and Lockheed Martin Advanced Technology Center; operations were enabled through the cooperation of the East Asian Observatory. When (some of) the data reported here were acquired, UKIRT was operated by the Joint Astronomy Centre on behalf of the Science and Technology Facilities Council of the U.K.

\section{REFERENCES}

Alexander D., Smail I., Bauer F., Chapman S., Blain A. W., Brandt W., Ivison R., 2005a, Nature, 434, 738

Alexander D., Bauer F., Chapman S., Smail I., Blain A., Brandt W., Ivison R., 2005b, ApJ, 632, 736

An F. X., et al., 2018, ApJ, 862, 101

Arancibia A. M., et al., 2018, A\&A, 620, A125

Aravena M., et al., 2016, ApJ, 833, 68

Archibald E. N., Dunlop J., Hughes D., Rawlings S., Eales S., Ivison R., 2001, MNRAS, 323, 417

Barger A., Cowie L., Sanders D., Fulton E., Taniguchi Y., Sato Y., Kawara K., Okuda H., 1998, Nature, 394, 248

Barger A., Cowie L., Richards E., 2000, ApJ, 119, 2092

Barger A., et al., 2014, ApJ, 784, 9

Béthermin M., De Breuck C., Sargent M., Daddi E., 2015, A\&A, 576, L9

Biggs A., et al., 2011, MNRAS, 413, 2314

Blain A. W., Smail I., Ivison R., Kneib J.-P., Frayer D. T., 2002, Phys. Reports, 369, 111

Blain A. W., Chapman S. C., Smail I., Ivison R., 2004, ApJ, 611, 52

Borys C., Smail I., Chapman S., Blain A., Alexander D., Ivison R., 2005, ApJ, 635, 853

Bothwell M., et al., 2013, MNRAS, 429, 3047

Brisbin D., et al., 2017, A\&A, 608, A15

Bruzual G., Charlot S., 2003, MNRAS, 344, 1000

Casali M., et al., 2007, A\&A, 467, 777

Casey C. M., et al., 2013, MNRAS, 436, 1919

Chabrier G., 2003, PASP, 115, 763

Chapman S. C., Blain A., Smail I., Ivison R., 2005, ApJ, 622, 772

Charlot S., Fall S. M., 2000, ApJ, 539, 718

Chen C.-C., et al., 2015, ApJ, 799, 194

Chen C.-C., et al., 2016, ApJ, 820, 82

Cimatti A., et al., 2008, A\&A, 482, 21

Connolly A., Szalay A., Dickinson M., Subbarao M., Brunner R., 1997, ApJL, 486, L11

Cooke E., et al., 2018, arXiv preprint arXiv:1805.05363

Coppin K., et al., 2008, MNRAS, 389, 45

Cowie L. L., Songaila A., Hu E. M., Cohen J., 1996, ApJ, 112, 839

Cowie L. L., Gonzalez-Lopez J., Barger A. J., Bauer F. E., Hsu L.-Y., Wang W.-H., 2018, ApJ, 865, 106

Cucciati O., et al., 2012, A\&A, 539, A31

Da Cunha E., Charlot S., Elbaz D., 2008, MNRAS, 388, 1595

Danielson A., et al., 2017, ApJ, 840, 78

Davis S. W., Laor A., 2011, ApJ, 728, 98

Dempsey J. T., et al., 2013, MNRAS, 430, 2534

Donley J., et al., 2012, ApJ, 748, 142

Drouart G., et al., 2014, A\&A, 566, A53 
Dunlop J. S., et al., 2017, MNRAS, 466, 861

Elvis M., et al., 1994, ApJS, 95, 1

Estrada-Carpenter V., et al., 2019, ApJ, 870, 133

Ferrarese L., Merritt D., 2000, ApJL, 539, L9

Franco M., et al., 2018, A\&A, 620, A152

Fujimoto S., Ouchi M., Ono Y., Shibuya T., Ishigaki M., Nagai H., Momose R., 2016, ApJ Supp., 222, 1

Furusawa H., et al., 2008, ApJS, 176, 1

Galametz A., et al., 2013, ApJS, 206, 10

Geach J., et al., 2017, MNRAS, 465, 1789

Gebhardt K., et al., 2000, ApJL, 539, L13

Genzel R., Baker A. J., Tacconi L. J., Lutz D., Cox P., Guilloteau S., Omont A., 2003, ApJ, 584, 633

Georgantopoulos I., Rovilos E., Comastri A., 2011, A\&A, 526, A46

González J. E., Lacey C., Baugh C., Frenk C., 2011, MNRAS, 413, 749

Gullberg B., et al., 2018, ApJ, 859, 12

Gültekin K., et al., 2009, ApJ, 698, 198

Hainline L. J., Blain A., Smail I., Alexander D., Armus L., Chapman S., Ivison R., 2011, ApJ, 740, 96

Hao C.-N., Xia X.-Y., Mao S.-D., Deng Z.-G., Wu H., 2008, Chinese J. Astron. Astrophys., 8, 12

Hatsukade B., et al., 2016, PASJ, 68

Hatsukade B., et al., 2018, PASJ, 70, 105

Hickox R. C., et al., 2012, MNRAS, 421, 284

Hill R., et al., 2018, MNRAS, 477, 2042

Hodge J., et al., 2013, ApJ, 768, 91

Hodge J., et al., 2016, ApJ, 833, 103

Hopkins P. F., Richards G. T., Hernquist L., 2007, ApJ, 654, 731

Hopkins P. F., Hernquist L., Cox T. J., Kereš D., 2008, ApJS, 175,356

Hughes D. H., et al., 1998, Nature, 394, 241

Ikarashi S., et al., 2015, ApJ, 810, 133

Ikarashi S., et al., 2017, ApJL, 849, L36

Ivison R., Smail I., Le Borgne J.-F., Blain A., Kneib J.-P., Bezecourt J., Kerr T., Davies J., 1998, MNRAS, 298, 583

Ivison R. J., et al., 2002, MNRAS, 337, 1

Ivison R. J., et al., 2007, MNRAS, 380, 199

Jarvis M. J., et al., 2012, MNRAS, 428, 1281

Johnson S. P., et al., 2013, MNRAS, 431, 662

Karim A., et al., 2013, MNRAS, p. stt196

Kocevski D. D., et al., 2018, ApJS, 236, 48

Koprowski M., Dunlop J., Michałowski M., Cirasuolo M., Bowler R., 2014, MNRAS, 444, 117

Kulkarni G., Worseck G., Hennawi J. F., 2018, arXiv preprint arXiv:1807.09774

Laird E. S., Nandra K., Pope A., Scott D., 2010, MNRAS, 401, 2763

Lilly S. J., Eales S. A., Gear W. K., Hammer F., Le Fevre O., Crampton D., Bond J. R., Dunne L., 1999, ApJ, 518, 641

Lusso E., et al., 2012, MNRAS, 425, 623

Magnelli B., et al., 2012, A\&A, 539, A155

Magorrian J., et al., 1998, ApJ, 115, 2285

McAlpine S., et al., 2019, arXiv preprint arXiv:1901.05467

McMullin J., Waters B., Schiebel D., Young W., Golap K., 2007, in Astronomical data analysis software and systems XVI. p. 127

Merloni A., 2004, MNRAS, 353, 1035

Michałowski M. J., Hayward C. C., Dunlop J. S., Bruce V. A., Cirasuolo M., Cullen F., Hernquist L., 2014, A\&A, 571, A75

Miettinen O., et al., 2017, A\&A, 606, A17

Morishita T., et al., 2018, arXiv preprint arXiv:1812.06980

Narayanan D., Hayward C. C., Cox T. J., Hernquist L., Jonsson P., Younger J. D., Groves B., 2010, MNRAS, 401, 1613

Oliver S., et al., 2012, MNRAS, 424, 1614

Pope A., Borys C., Scott D., Conselice C., Dickinson M., Mobasher B., 2005, MNRAS, 358, 149
Riechers D. A., et al., 2011, ApJL, 733, L12

Rieke G. H., Alonso-Herrero A., Weiner B., Pérez-González P. G., Blaylock M., Donley J., Marcillac D., 2009, ApJ, 692, 556

Sanders D., Soifer B., Elias J., Madore B., Matthews K., Neugebauer G., Scoville N., 1988, ApJ, 325, 74

Scudder J. M., Oliver S., Hurley P. D., Griffin M., Sargent M. T., Scott D., Wang L., Wardlow J. L., 2016, MNRAS, 460, 1119

Simpson J., et al., 2012, MNRAS, 426, 3201

Simpson J. M., et al., 2014, ApJ, 788, 125

Simpson J., et al., 2015a, ApJ, 799, 81

Simpson J., et al., 2015b, ApJ, 807, 128

Simpson J., et al., 2017, ApJ, 839, 58

Smail I., Ivison R., Blain A., 1997, ApJL, 490, L5

Smail I., Chapman S., Blain A., Ivison R., 2004, ApJ, 616, 71

Smolčić V., et al., 2012, A\&A, 548, A4

Stach S. M., et al., 2018, ApJ, 860, 161

Swinbank A., Smail I., Chapman S., Blain A., Ivison R., Keel W. C., 2004, ApJ, 617, 64

Swinbank A., Chapman S., Smail I., Lindner C., Borys C., Blain A., Ivison R., Lewis G., 2006, MNRAS, 371, 465

Swinbank A., et al., 2012, MNRAS, 427, 1066

Swinbank A., et al., 2014, MNRAS, 438, 1267

Tacconi L. J., et al., 2006, ApJ, 640, 228

Takata T., Sekiguchi K., Smail I., Chapman S. C., Geach J., Swinbank A., Blain A., Ivison R., 2006, ApJ, 651, 713

Toft S., et al., 2014, ApJ, 782, 68

Umehata H., et al., 2014, MNRAS, 440, 3462

Walter F., et al., 2016, ApJ, 833, 67

Wang W.-H., Cowie L. L., Barger A. J., Williams J. P., 2011, ApJL, 726, L18

Wang S., et al., 2013, ApJ, 778, 179

Weiß A., et al., 2009, ApJ, 707, 1201

Weiß A., et al., 2013, ApJ, 767, 88

Wilkinson A., et al., 2017, MNRAS, 464, 1380

Younger J. D., et al., 2007, ApJ, 671, 1531

Younger J., et al., 2008, MNRAS, 387, 707

da Cunha E., et al., 2015, ApJ, 806, 110

This paper has been typeset from a $\mathrm{T}_{\mathrm{E}} \mathrm{X} / \mathrm{LAT}_{\mathrm{E}} \mathrm{X}$ file prepared by the author. 